\title{
Diversity and distribution of palms (Arecaceae) in Assam, India
}

\author{
Selim Mehmud ${ }^{1 *}$, Himu Roy $^{1}$ \\ 1 Department of Botany, Cotton University, Panbazar, Guwahati-781001, Assam, India \\ * Corresponding author, mehmudselim@gmail.com
}

\begin{abstract}
We present new information on the diversity and distribution of palms, family Arecaceae, in Assam state, India. The family is represented in the state by 44 species belonging to 19 genera. Our study is based on surveys, a literature review, and a herbarium examination. Identification keys to genera and species, diagnostic characters, vernacular names, and updated information on the distribution, flowering, and fruiting of both wild and cultivated palm species occurring in Assam are provided.
\end{abstract}

Keywords

Barak Valley, Brahmaputra Valley, documentation, identification, Monocot, Palmae

Academic editor: Anzar Khuroo | Received 10 February 2020 | Accepted 28 Nov 2020 | Published 13 January 2021

Citation: Mehmud S, Roy, H (2021) Diversity and distribution of palms (Arecaceae) in Assam, India. Check List 17 (1): 69-93. https://doi.org/ $10.15560 / 17.1 .69$

\section{Introduction}

The family Arecaceae (palms) is represented by over 2500 species globally and have a variety of growth forms such as shrubs, lianas, and trees (Muscarella et al. 2020). Palms are commonly distributed in tropics and subtropics and considered to be the economically important plants, second only to the Poaceae (grasses) (Renuka and Sreekumar 2012; Qureshimatva et al 2020). Palms are used for food, medicine, household materials, furniture, and other purposes (Johnson 1998).

In India, the earliest account of the family can be traced back to the work of van Rheede tot Draakestein's (1678) Hortus Indicus Malabaricus. Assam, in the northeastern region of India, is known for its rich biodiversity (Bora and Bhattacharyya 2017). Griffith (1850) reported 19 species, and Blatter (1926) reported 28 species and one variety from Assam. The family Arecaceae was excluded from the Flora of Assam (Kanjilal et al. 1934-1940). Detailed taxonomic studies on the family are still unavailable for Assam (Rao and Verma 1970, 1972, 1973, 1974 and 1976; Jain and Hajra 1975; Rahman 2007; Bora et al. 2012; Mehmud and Roy 2020, 2020a, 2020b, 2020c). Renuka and Sreekumar (2012) reported 105 species belonging to 22 genera from India, whereas Barooah and Ahmed (2014) listed 36 species belonging to 18 genera from Assam.

In this backdrop, we investigated the taxonomic diversity and local distribution of Arecaceae in Assam and focus on providing taxonomic details of the species, diversity, and distribution along with their local uses.

\section{Methods}

Study area. Assam is a northeastern India state having an area of over $78,000 \mathrm{~km}^{2}$. The state receives an annual rainfall ranging from $178-305 \mathrm{~cm}$ and experience an average humidity of $83 \%$ (Baishya et al. 2015). 
Field survey and data collection. We made extensive field surveys to collect palms from August 2017 to February 2020. We conducted 68 trips, either as single-day or multi-day (5-15 day) expeditions throughout reserve forests, wildlife sanctuaries, and non-protected forest areas. We recorded details on distribution, flowering, fruiting, and the vernacular name for each species and collected voucher specimens. Geographic coordinates were recorded using a Garmin etrex 10 GPS receiver.

Specimen collection, identification, and herbarium preparation. The collection of specimens and their preparation for the herbarium followed standard procedures (Jain and Rao 1977; Dransfield 1986). We identified species using the literature (Griffith 1845; Beccari 1908, 1911; Basu 1992; Basu and Chakraverty 1994; Barrow 1998; Dowe 2009; Renuka et al. 2010; Renuka and Shreekumar 2012; Henderson 2007, 2009, 2020) by comparing our specimens with specimens in herbaria of Eastern Regional Centre of Botanical Survey of India (acronym: ASSAM), Central National Herbarium of Botanical Survey of India (acronym: CAL), Gauhati University Botany Herbarium (acronym: GUBH), Meise Botanic Garden Herbarium (acronym: BR) and online herbarium Kew Herbarium Catalogue (acronym: K), New York Botanical Herbarium (acronym: NYBG), Meuseum National d' Histoire Naturelle (acronym: P) and Palm Web (2019). Species names used here follow the accepted names in The Plant List (2020). Information related to vernacular names and uses of different species was documented during the field survey by consulting local people. Based on the presence and absence of scales on fruits, the species are categorized into Group A and Group B respectively and site of their collection (voucher specimens) is shown in Figure 1.

Voucher specimens were submitted to ASSAM. Other specimens are kept in the herbarium of the Department of Botany, Cotton University (abbreviated CH here).

\section{Results}

We recorded 44 species belonging to 19 genera of Arecaceae from Assam. The genus Calamus, with 15 species, is the most diverse, followed by Pinanga, Livistona, and Wallichia, each with three species. Areca, Caryota, Dypsis, Phoenix, and Salacca each presented two species, and other genera such as Arenga, Borassus, Cocos, Corypha, Elaeis, Licuala, Plectocomia, Ptychosperma, Rhapis, and Roystonea were represented by single species. Two species, Corypha umbraculifera and Salacca wallichiana, are recorded for the first time from Assam.

\section{Key to the genera}

1a Fruit scaly.

1b Fruit non-scaly 4

2a Palms acaulescent Salacca 3a Fruit scales margin hairy/wooly; leaflets abaxially white Plectocomia

3b Fruit scales margin not hairy/wooly; leaflets abaxially green

Calamus

4a Leaf palmate ..................................................... 5

4b Leaf pinnate ..................................................... 9

5a Stem ca $50 \mathrm{~cm}$ in diameter................................. 6

5b Stem less than $50 \mathrm{~cm}$ in diameter ....................... 7

6a Hepaxanthic palm; petiole spiny ............. Corypha

6b Pleonanthic palm; petiole serrate or dentete.....

Borassus

7a Rhizomatous; petiole without spine ........ Rhaphis

7b Non-rhizomatous; petiole with spines ................. 8

8a Flowers ca 2 cm long; fruits red ................ Licuala

8b Flowers ca $0.6 \mathrm{~cm}$ long; fruits bluish to red

Livistona

9a Palms with bipinnate leaf .......................... Caryota

9b Palms with unipinnate leaf ................................10

10a Seeds deeply channeled ................. Ptychosperma

10b Seeds shallowly channeled .................................11

11a Palms armed ....................................................... 12

11b Palms unarmed .............................................. 13

12a Petiole with acanthophylls; leaflets tip sharp .........

Phoenix

12b Petiole without acanthophylls; leaflets tip not sharp Elaeis

13a Stem covered by black fibre ............................. 14

13b Stems lacks black fibre ...................................... 15

14a Leaflets in one plane; auricle ca $3 \mathrm{~cm}$....... Arenga

14b Leaflets in one or two plane; auricle rarely ca $6 \mathrm{~mm}$

Wallichia

15a Fruits large ca $15 \mathrm{~cm}$ in diameter ................ Cocos

15b Fruits less than $15 \mathrm{~cm}$ in diameter.....................16

16a Stem ca $50 \mathrm{~cm}$ in diameter; pistillate and staminate flowers in different inflorescences....

Roystonea

16b Stem less than $50 \mathrm{~cm}$ in diameter; pistillate and staminate flowers in same inflorescence. .17

17a Pistillate flowers at the base of rachilla and staminate flowers at apex Areca

17b Pistillate flower in between two staminate flowers 18

18a Stems ca 8-32 cm in diameter ................... Dypsis

18b Stems ca $2.5 \mathrm{~cm}$ in diameter................... Pinanga

Key to the species of Areca

1a. Solitary palm, ca $20 \mathrm{~m}$ tall; stamens 6

Areca catechu

1b Clustered palm, ca 3-5 m tall; stamens 3

Areca triandra

\section{Areca catechu $\mathrm{L}$.}

Diagnostic characters. Solitary, erect, unarmed palm, $20 \mathrm{~m}$ tall; internodes grey-black, $10-16 \mathrm{~cm}$ long and 5-8 $\mathrm{cm}$ in diameter. Leaf pinnate, $1.5-3 \mathrm{~m}$ long; sheath 1-1.5 



Figure 1. Map of the study area Assam, India. Species records plotted on the map according with the legend. 
m long; crownshaft green. Inflorescence branched, interfoliolar below the leaf; spathe 60-72 cm long, light yellowish, tip acute; rachis with many rachilla, 30-35 cm long; pistillate flowers at base and staminate at terminal. Staminate flowers with 6 stamens. Fruit ovoid, red, $3 \mathrm{~cm}$ long, and $2.5 \mathrm{~cm}$ wide.

Flowering and fruiting. Throughout the year.

Distribution. Throughout Assam.

Vernacular names. Tamul, Gua (Assamese); Gua, Supari (Bengali).

Uses. As food and masticator (nut), broom (leaf), disposable dish (leaf sheath), hut (mature dry stem).

Material examined. INDIA • Assam, Kamrup Metro, Guwahati, Chandmari; 26¹1'44"N, 091 $46^{\circ} 57^{\prime \prime} \mathrm{E}$; 06 May 2018; S. Mehmud leg.; CH.

\section{Areca triandra Roxb. ex Buch.-Ham.}

Diagnostic characters. Clustered, unarmed, 3-5 m tall palm; stem green, internodes $10-16 \mathrm{~cm}$ long, and $3-4 \mathrm{~cm}$ in diameter. Leaf pinnate, $1-1.5 \mathrm{~m}$ long; crownshaft 30-45 cm long; leaflets opposite to subopposite, 14-30 per side, $42 \mathrm{~cm}$ long, entire, acute to acuminate. Spathe green, oblong, $30 \mathrm{~cm}$ long, and $6 \mathrm{~cm}$ wide. Inflorescence branched, rachillae green, $20-30 \mathrm{~cm}$ long; pistillate flowers at base and staminate flowers at apex. Staminate flowers with 3 stamens. Fruits red, ellipsoid, 3-4 $\mathrm{cm}$ long, and ca $1.5 \mathrm{~cm}$ wide.

Flowering and fruiting. March to September and March to April, respectively.

Distribution. Common in Assam; wild or ornamental; prefers open fields and hilly areas.

Vernacular names. Ram Gua (Assamese); Garo Tamul (Garo, Assamese).

Uses. Ornamental. As masticator (nut).

Material examined. INDIA • Assam, Mikir Hills district (Karbi Anglong), Bokajan; 21 Jun. 1963; D. B. Deb leg.; ASSAM 34697. • Assam, Bongaigaon, Thakurani Para, Kakoijana Area; 2625'4"N, 090³9'04"E; alt. 52 m; 25 Mar. 2018; S. Mehmud leg.; CH, ASSAM 96213.

\section{Arenga westerhoutii Griff.}

Figure 2A

Diagnostic characters. Solitary, unarmed, $12 \mathrm{~m}$ tall woody tree; trunk $25-30 \mathrm{~cm}$ in diameter. Leaf pinnate, erect, 4-5.5 m long; petiole green, with black fibre at base. Leaflets auriculate, alternate, 100-130 per side, 28-90 cm long, 5-8 cm wide, linear, adaxially dark green, abaxially silvery white with brown indumentums and prominent midvein, auricle ca $3 \mathrm{~cm}$ long and apex serrated.

Distribution. Cachar district; hilly areas.

Material examined. INDIA - Arunachal Pradesh, Dibang Valley, Angolin; 720 m; 27 Aug. 2000; M. Bhaumik leg.; ASSAM 64953. • Assam, Cachar, Borail WLS; 17 Jul. 2011; Hussain Ali Barbhuiyan leg.; ASSAM 85989. • Assam, Cachar, Balicherra, Haflong Road; 2459'24.6"N, 092 45'10.7"E; alt. 83 m; 09 Nov. 2018; S. Mehmud leg.; CH, ASSAM 96214.

\section{Borassus flabellifer L.}

Diagnostic characters. Solitary, erect, 20-25 m tall tree; trunk grey-black, ca $50 \mathrm{~cm}$ in diameter, smooth or covered with dry petiole. Leaf costapalmate, 3-3.8 m long; petiole 1.5-1.8 m long, green or yellowish, margin black and serrated. Leaflets up to 70 in number, apices bifid, ca $10 \mathrm{~cm}$ long, acute. Staminate inflorescence arise from leaf base, ca $1 \mathrm{~m}$ long, with 9-11 alternate branches; bracts ca $50 \mathrm{~cm}$ long, tubular; rachis $40-46 \mathrm{~cm}$ long, with rachillae $2-4$, ca $30 \mathrm{~cm}$. Female inflorescence ca $90 \mathrm{~cm}$ long; bracts ca $40 \mathrm{~cm}$; rachillae ca $23 \mathrm{~cm}$. Fruits globose, black, ca $15 \mathrm{~cm}$ in diameter.

Flowering and fruiting. March to May and June to November, respectively

Distribution. Common in Assam; plain areas.

Vernacular names. Tal (Assamese).

Uses. As food (fruit), firewood (leaf, inflorescence), hut (leaf).

Material examined. INDONESIA - East Java, Pasuruan, Purwodadi; alt. 300 m; 27 Nov. 1988; Harini leg.; NYBG 02320483. INDIA • 1 §̧; Assam, Morigaon, Thengbhanga; $26^{\circ} 12^{\prime} 30^{\prime \prime} \mathrm{N}, 092^{\circ} 03^{\prime} 12^{\prime \prime} \mathrm{E}$; alt. $1 \mathrm{~m}$; 08 Apr. 2018; S. Mehmud leg.; CH, ASSAM 96215.

\section{Key to the species of Calamus}

1a Climbing rattan, climbing organ flagella or cirri ... 2

1b Non climbing rattan, climbing organs absent ........

Calamus erectus

2a Leaf cirriate; inflorescence without flagella ........ 3

2b Leaf ecirriate; inflorescence with flagella .......... 6

3a Inflorescence boat shaped

Calamus melanochaetes

3b Inflorescence not boat shaped ............................ 4

4a Leaflets ovate or lanceolate, arrange in groups ... 5

4b Leaflets lanceolate, arrange regularly.

Calamus nambariensis

5a Leaflets ovate-lanceolate, paired; knee wrinkled .. Calamus palustris

5b Leaflets lanceolate, in groups of 2-4; knee smooth Calamus inermis

6a Leaflets grouped .............................................. 7

6b Leaflets regular .............................................. 10

7a Leaflets not more than 8 per side of the rachis..... 8

7b Leaflets more than 8 per side of the rachis ........ 9

8a Ocrea conspicuous, bristly, ca $5 \mathrm{~mm}$ long

Calamus meghalayensis

8b Ocrea inconspicuous, spiny, ca 4-5 mm long ........ Calamus kingianus

9a Petiole with linear spines, ca $3 \mathrm{~cm}$ long; fruits green Calamus floribundus

9b Petiole with triangular spines, ca $7 \mathrm{~mm}$ long; fruits red

Calamus gracilis

10a Stems solitary; above 1200 m elevation; leaf with vestigial cirri

Calamus acanthospathus 


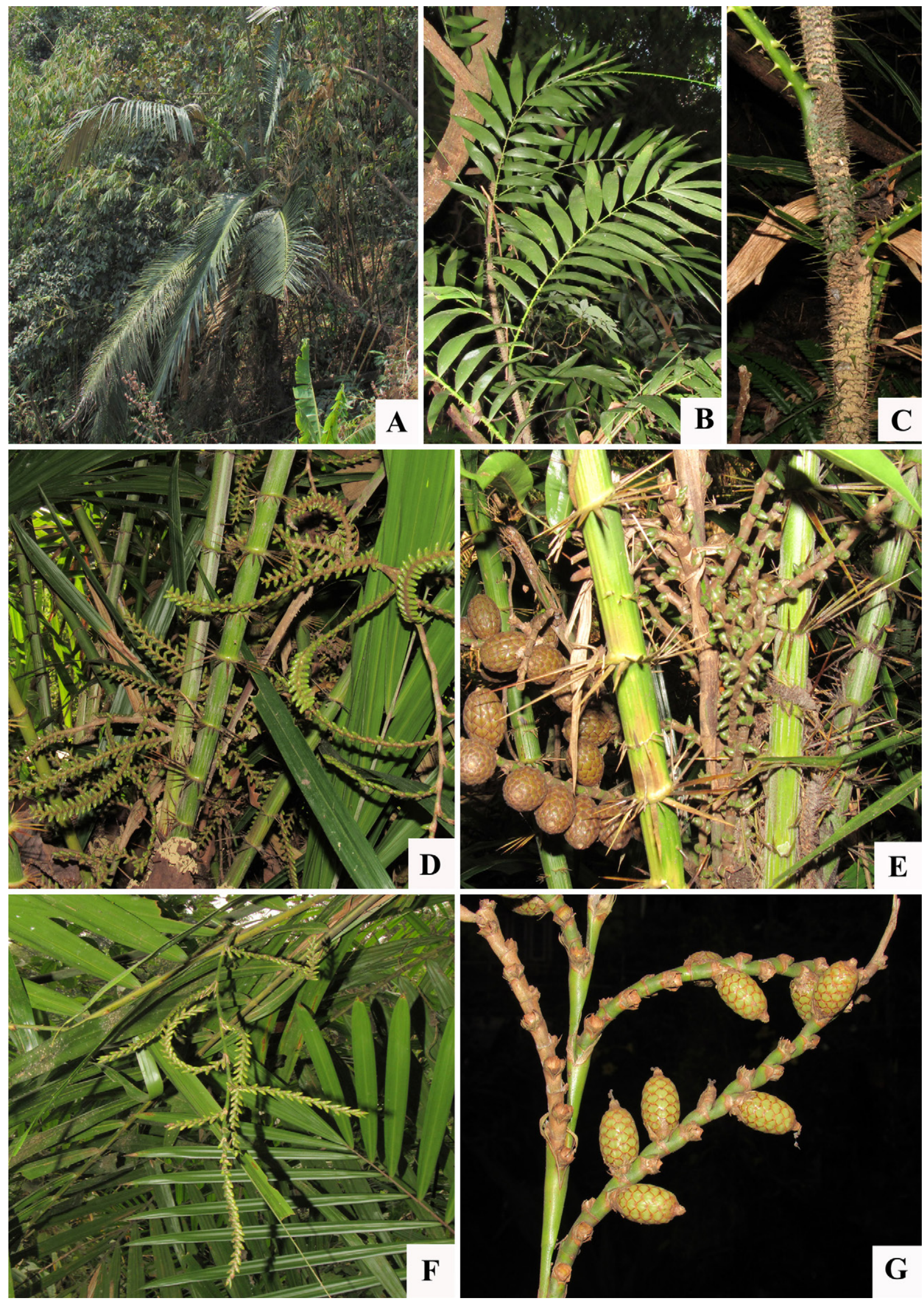

Figure 2. Palms (Arecaceae) in Assam, India. A. Arenga westerhoutii, habit. B, C. Calamus acanthospathus: (B) habit; (C) stem. D, E. Calamus erectus: (D) petiole with staminate inflorescence; $(\mathbf{E})$ pistillate inflorescence and fruiting. F, G. Calamus flagellum: (F) staminate inflorescence; (G) fruiting branch. 
10b Stems clustered; below 1200 m elevation; leaf without vestigial cirri 11

11a Leaflets terminally connate ... Calamus flagellum

11b Leaflets terminally free.... .. 12

12a Ocrea ca $13 \mathrm{~cm}$ long; partial inflorescence bracts ca 20-30 cm long Calamus guruba

12b Ocrea less than $13 \mathrm{~cm}$; partial inflorescence bracts less than 20-30 cm long

13a Ocrea bristly, ca $3 \mathrm{~cm}$; staminate rachillae scorpoid and bracteole up to half portion

Calamus leptospadix

13b Ocrea non bristly; staminate rachillae not scorpoid and bracteole at base.... 14

14a Stem with brown indumentums; partial inflorescence terminated by a long rachilla

Calamus henryanus

14b Stems with silvery-white indumentums; partial inflorescence not terminated by a long rachilla

Calamus tenuis

\section{Calamus acanthospathus Griff.}

Figure 2B, C

Diagnostic characters. Stems solitary, $10 \mathrm{~m}$ long; stems with leaf sheath $2 \mathrm{~cm}$ in diameter; spines linear, triangular $0.3-1 \mathrm{~cm}$ long. Knee $3 \mathrm{~cm}$ long, green, armed by ca $2 \mathrm{~mm}$ long spines; ocrea ca 5-6 mm long, spiny. Leaf 1 $\mathrm{m}$ long, ecirriate; petiole with single or paired spines ca $1 \mathrm{~cm}$; leaflets regular, 8-13 per side, alternate, lanceolate to ensiform, $30 \mathrm{~cm}$ long, acuminate, terminally free, veins 7 in numbers, vestigial cirri ca $2 \mathrm{~mm}$. Flagella 2.5 $\mathrm{m}$ long, grapnel spines ca $6 \mathrm{~mm}$ long.

Distribution. Dima Hasao district; in dense forest.

Uses. Domestic uses (cane).

Material examined. INDIA - Assam, Khasi \& Jaintia Hills, Jowai; alt. 4500 feet; 6 Jan. 1916; Upendranath Kanjilal leg.; ASSAM 30954, CAL 493911. • Assam, Dima Hasao, Jatinga; $25^{\circ} 06^{\prime} 24.2^{\prime \prime} \mathrm{N}, 093^{\circ} 02^{\prime} 55.8^{\prime \prime} \mathrm{E}$; alt. 1203 m; 14 Nov. 2019; S Mehmud leg.; CH, ASSAM 96216.

\section{Calamus erectus Roxb.}

Figure 2D, E

Diagnostic characters. Stems clustered, non climbing, erect, ca $4 \mathrm{~m}$ tall; petioles with linear spines, ca $4 \mathrm{~cm}$ long and arranged in whorls. Internodes ca $7 \mathrm{~cm}$ long, and $4-6 \mathrm{~cm}$ in diameter, green. Leaf pinnate, ecirriate, ca 3.5 m long; ocrea spiny. Leaflets 30-46 per side, lanceolate, 40-65 cm long, ca $3 \mathrm{~cm}$ wide, acuminate, veins 3 in numbers, terminal leaflets connate at base. Inflorescence ca $1 \mathrm{~m}$ long, flagella absent; partial inflorescence up to 10 in number, $20-25 \mathrm{~cm}$ long; staminate rachillae 6-9 cm long; pistillate rachillae 10-15 cm long with dyad flowers. Fruits ellipsoid, brown, 3-3.5 cm long, scales in 12 vertical rows, grooved.

Flowering and fruiting. July to December.

Distribution. Cachar, Dima Hasao and East Karbi Anglong districts; prefers shaded areas.
Vernacular names. Jeng Bet (Assamese).

Uses. As handle for knives (stem).

Material examined. INDIA - Meghalaya, Pynursla; $25^{\circ} 10^{\prime} 51.10^{\prime \prime} \mathrm{N}, 091^{\circ} 53^{\prime} 34.27^{\prime \prime} \mathrm{E}$; 30 Mar. 2012; Ramesh Kumar \& party leg.; ASSAM 91796. • NEFA (North-East Frontier Agency), Subansiri, New Head Quarters; alt. 241 m; 26 Sep. 1959; G. Panigrahi leg.; ASSAM 20108. - 1 đ̊;; Assam, Karbi Anglong (Mikir Hills), Kalioni Reserve Forest; 06 Feb. 1977; S. K. Borthakur leg.; ASSAM 76680. • 19, $80 \mathrm{~cm}, \mathrm{~F}$; Assam, Cachar, Bhuban Hill;

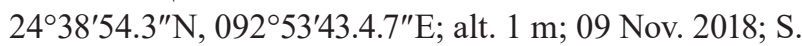
Mehmud leg.; CH, ASSAM 96217. • 1 ऊ’; same collection data as per preceding; S. Mehmud leg.; CH.

\section{Calamus flagellum Griff. ex Mart.}

Figure 2F, G

Diagnostic characters. Stems clustered, climbing, ca $12 \mathrm{~m}$ long, ca $2.5 \mathrm{~cm}$ in diameter. Leaf sheath with brown or grey indumentums; spines linear $4.5-5 \mathrm{~cm}$; flagella ca $4.5 \mathrm{~m}$ long. Knee $6 \mathrm{~cm}$ long; ocrea spiny, 2-3 cm long. Leaf ecirriate, 2.5-3 m long; leaflets regular, 25-33 per side, opposite to alternate, linear, serrated, acuminate, $66-74 \mathrm{~cm}$ long and ca $3.5 \mathrm{~cm}$ wide, veins 3 in number, terminal leaflets connate. Inflorescences ca $6 \mathrm{~m}$ long, flagella 1.5-2 m long; partial inflorescence 5-6 in number, 40-70 cm long; rachillae 10-40 cm long; pistillate rachillae with dyad flowers. Fruits ellipsoid, $2.5-2.8 \mathrm{~cm}$ long, scales in 12 vertical rows, greenish yellow.

Flowering and fruiting. October to March and February to March, respectively.

Distribution. Common; near streams.

Vernacular names. Raidang (Assamese); Rai (Dimasha). Uses. Furniture and other house hold products (cane), food (apical stem).

Material examined. INDIA - Arunachal Pradesh, Dibang Valley, near Jambupani way to Deshali from Hunli; alt. 700 m; 25 Feb. 2004; M. Bhaumik leg.; ASSAM 64981 , 64982. - Assam, Nizamghat; alt. 450 ft; 05 Dec. 1913; Upendranath Kanjila leg.; ASSAM 30917. - 1 今ं; Assam, Cachar, Dwarband; 2432'25"N, $092^{\circ}$ 44'37"E; alt. 12 m; 19 Nov. 2018; S. Mehmud leg.; CH, ASSAM 96218. 1 + ; Assam, Tinsukia, Margherita; $27^{\circ}$ $14^{\prime} 22.8^{\prime \prime} \mathrm{N}, 095^{\circ} 35^{\prime} 02.7^{\prime \prime} \mathrm{E}$; alt. $113 \mathrm{~m}$; 16 Feb. 2019; S. Mehmud leg.; CH, ASSAM 96219.

\section{Calamus floribundus Griff.}

Figure 3A, B

Diagnostic characters. Stems clustered, climbing, ca 5 $\mathrm{m}$ long; with leaf sheath ca $1.5-2 \mathrm{~cm}$ in diameter; spines black brown, linear, 1-3 cm long; flagella ca $2 \mathrm{~m}$ long. Knee ca $2 \mathrm{~cm}$ long, green, spiny; ocrea spiny or bristly, ca $6 \mathrm{~mm}$ long. Leaf pinnate, ecirriate, ca $1.5 \mathrm{~m}$ long; leaflets $10-17$ per side, $20-41 \mathrm{~cm}$ long and $2.5-5 \mathrm{~cm}$ wide, alternate to opposite, single or in groups, obovate to lanceolate, serrated, acute, veins 3 , terminally connate. Staminate inflorescence $1.5-2 \mathrm{~m}$ long; flagella $0.8-0.9 \mathrm{~m}$ long; partial inflorescences $10-22 \mathrm{~cm}$ long; rachillae 2-5 

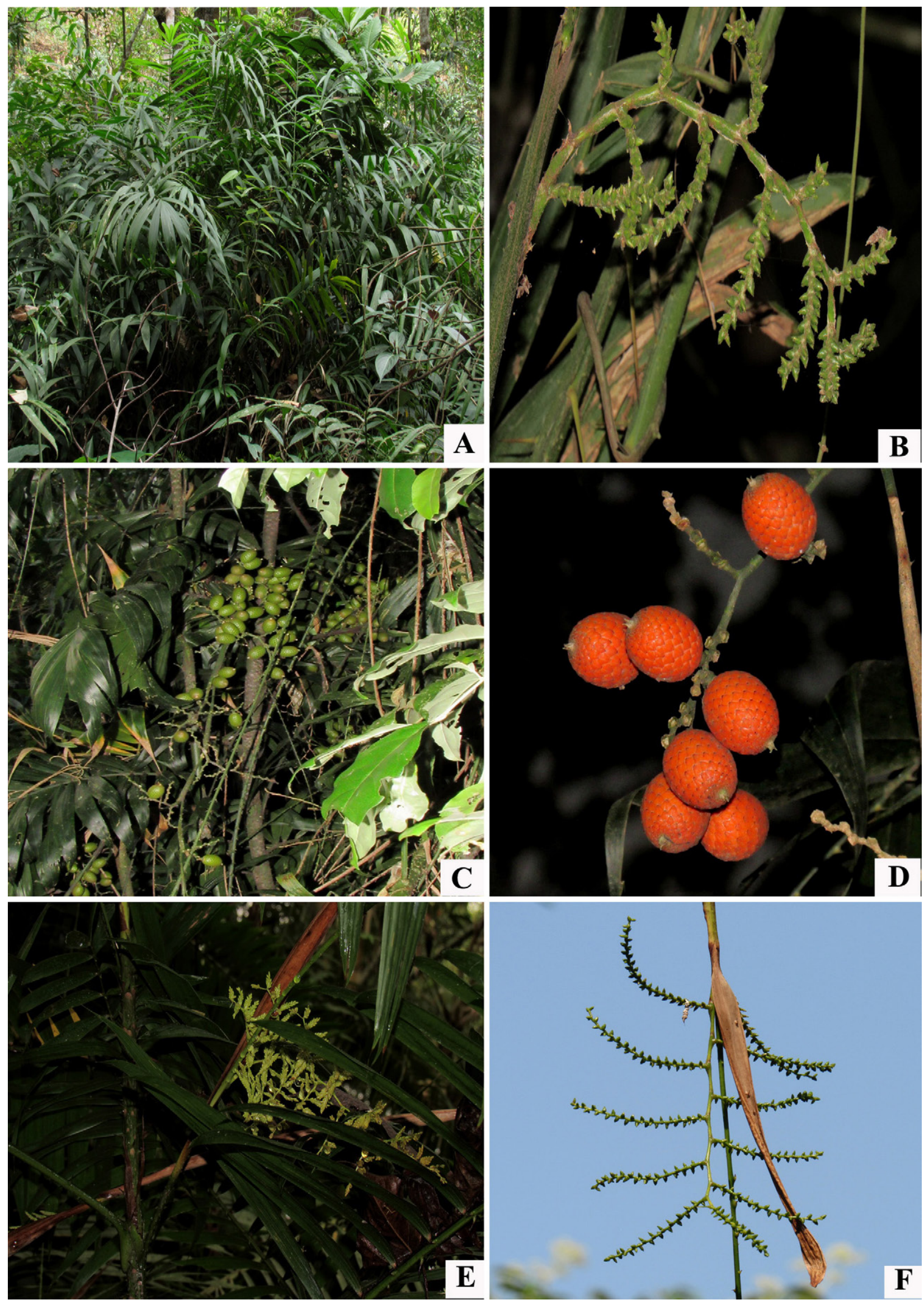

Figure 3. Palms (Arecaceae) in Assam, India. A, B. Calamus floribundus: (A) habit; (B) pistillate partial inflorescence. C, D. Calamus gracilis: (C) habit with immature fruit; (D) ripe fruit. E, F. Calamus guruba: (E) habit and staminate inflorescence; (F) pistillate partial inflorescence. 
$\mathrm{cm}$ long. Pistillate inflorescence 2-3.5 m long; flagella 1.5-2 m long; partial inflorescences $18 \mathrm{~cm}$ long; rachillae alternate, 5-7 cm long with dyad. Fruits globose to ellipsoid, ca $1.3 \mathrm{~cm}$ long, green, scales in 15 vertical rows, grooved.

Flowering and fruiting. December to April, May to June respectively.

Distribution. Common; in waterlogged open areas.

Vernacular names. Lejai (Assamese); Pani or Tenga Bet (Rabha).

Uses. As binding material (cane), masticator (fruits).

Material examined. INDIA • Arunachal Pradesh, Namdapha, Miao Forest; 11 Jun. 1982; J. Joseph leg.; ASSAM 66478. • Assam, Khasi \& Jaintia Hill, Cherapunjee to Mawmluh; 27 Sep. 1950; G. Panigrahi leg.; CAL. •

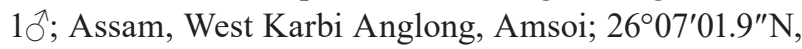
092 26'25.4"E; alt. 126 m; 24 Feb. 2018; S. Mehmud leg.; ASSAM 96221. • 1\%; Assam, West Karbi Anglong, Nelie Umapanai Khenduli Tapat Road; $26^{\circ} 02^{\prime} 24.9^{\prime \prime} \mathrm{N}$, 092 16'2.9"E; alt. 74 m; 30 Mar. 2018; S. Mehmud leg.; CH, ASSAM 96220. • 1ठ̄; Assam, Cachar, Daulashal; $24^{\circ} 26^{\prime} 16.5^{\prime \prime} \mathrm{N}, 092^{\circ} 49^{\prime} 20.8^{\prime \prime} \mathrm{E}$; alt. 03 m; 15 Nov. 2018; S. Mehmud leg.; $\mathrm{CH}$.

\section{Calamus gracilis Roxb.}

Figure 3C, D

Diagnostic characters. Stems clustered, climbing, 7-10 $\mathrm{m}$ long, with leaf sheath $2-2.3 \mathrm{~cm}$ in diameter; indumentums brown; spines triangular, upward pointing, ca $2 \mathrm{~mm}$ long; flagella $2.2-3 \mathrm{~m}$ long. Knee ca $2 \mathrm{~cm}$ long, wrinkled, spiny; ocrea ca $5 \mathrm{~mm}$ long, unarmed. Leaf ecirriate, 30-52 cm long; petiole $0.8-1.2 \mathrm{~cm}$ long. Leaflets grouped, opposite, $16-18$ per side, ca $26 \mathrm{~cm}$ long and ca $3 \mathrm{~cm}$ wide, lanceolate to ensiform, serrated, acuminate, tip bent and facing downward, terminally paired, veins 3. Staminate inflorescence ca $90 \mathrm{~cm}$ long, flagella ca 6-9 cm long; rachillae alternate, flowers globose. Pistillate inflorescence ca $1 \mathrm{~m}$ long; partial inflorescence 13-17 cm long; rachillae 3-5 cm long. Fruits ellipsoid, ca $2.5 \mathrm{~cm}$ long, orange-red, scales in 20 vertical rows.

Flowering and fruiting. May.

Distribution. Cachar and Dima Hasao districts; prefer hilly areas.

Vernacular names. Suli Bet (Assamese).

Uses. Binding material for preparation of traditional trays locally known as "dola" and "saloni" (cane).

Material examined. INDIA • Assam, Sibsagar, Kalioni; alt. 340ft; Upendranath Kanjilal leg.; ASSAM 30961, ASSAM 40720. • Assam, Cachar, Daulashal;

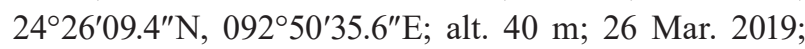
S. Mehmud leg.; CH. • Assam, Dima Hasao, Jatinga; 2507'56"N, 09301'17.8"E; alt. 671m; 12 Nov. 2019; S. Mehmud leg.; CH, ASSAM 96222.

\section{Calamus guruba Buch-Ham.}

Figure 3E, F

Diagnostic characters. Stems clustered, climbing, 5-8 $\mathrm{m}$ long, with leaf sheath $2-3 \mathrm{~cm}$ in diameter; spines triangular, ca $1.5 \mathrm{~cm}$ long; flagella $1.5-2 \mathrm{~m}$ long. Leaf pinnate, ecirriate, ca $1.3 \mathrm{~m}$ long; leaflets regular, 30-40 per side, elliptic to lanceolate, serrated, acuminate, 11$34 \mathrm{~cm}$ long, 0.7-2.5 cm wide and terminally free. Knee green, 3-3.5 cm long; ocrea 8-18 cm long. Inflorescence ca $3.5 \mathrm{~m}$ long; partial inflorescence covered by $20-30 \mathrm{~cm}$ long bract; flagella ca $2 \mathrm{~m}$ long; staminate partial inflorescences 11-25 cm long, rachillae 0.7-2 cm long; pistillate partial inflorescences $15-17 \mathrm{~cm}$ long, rachillae 7-11 $\mathrm{cm}$ long, with dyad flowers. Fruits globose, brown, ca 1 $\mathrm{cm}$ long, scales in 18 vertical rows.

Flowering and fruiting. November to February and January to February, respectively.

Distribution. Cachar, Darrang, Kamrup Rural, Kamrup Metro, Golaghat, Hailakandi, Jorhat, East Karbi Anglong, West Karbi Anglong, Karimganj districts; prefer open plain and sun exposed areas.

Vernacular names. Bhat Bet, Khagori Bet (Assamese); Sundi Bet (Bengali, Dimasha).

Uses. In fishing equipment (cane).

Material examined. INDIA - 1 ; Naga Hill, Rangapaha; 22 Nov. 1964; M. M. Srinivasan leg.; ASSAM 30960. • 1 đ̇; Assam, Cachar, Ramprosadpur; Feb. 1944; D. F. O. leg.; ASSAM. • Assam, Sibsagar, Disoi Valley; alt. 350ft; 27 Apr. 1913; Upendranath Kanjilal leg.; ASSAM 30955. • 1; Assam, Kamrup Rural, Barkhuli, Ukiam; 2552'14.2"N, 091²1'11" E; alt. 66 m; 04 Mar. 2018; S. Mehmud leg.; CH, ASSAM 96223. •1ð̄; Assam, Karimganj, Fanairbond; $24^{\circ} 25^{\prime} 53^{\prime \prime} \mathrm{N}, 092^{\circ} 25^{\prime} 55^{\prime \prime} \mathrm{E}$; alt. 03m; 10 Jan. 2019; S. Mehmud leg.; CH, ASSAM 96224.

\section{Calamus henryanus Becc.}

Figure 4A, B

Diagnostic characters. Stems clustered and climbing, ca $8 \mathrm{~m}$ long; leaf sheath with brown indumentums and triangular spines, ca. $2 \mathrm{~cm}$ long; flagella ca $1.5 \mathrm{~m}$ long. Knee glabrous or spiny, ca $2.5 \mathrm{~cm}$ long; ocrea ca $2 \mathrm{~mm}$ long. Leaf ecirriate, ca $1 \mathrm{~m}$ long; leaflets regular, lanceolate; easily distinguishable by its unique partial inflorescence up to $23 \mathrm{~cm}$ long where length of rachillae gradually decrease from base to apex but terminal rachilla is comparatively longer than others and the neuter flower with pedicel.

Flowering. November to March.

Distribution. Cachar district (Mehmud and Roy 2020a). Uses. As binding material (cane).

Material examined. INDIA• 2 $\hat{O}^{\hat{*}}$; Assam, Cachar, Bhuban Hill; 243' $41.6^{\prime \prime} \mathrm{N}, 093^{\circ} 00^{\prime} 52^{\prime \prime} \mathrm{E}$; alt. $704 \mathrm{~m}$; 10 Nov. 2018; S. Mehmud leg.; CH, ASSAM 59113. 2ᄋ; Assam,

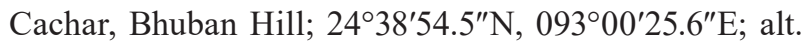
112 m; 23 Mar. 2019; S. Mehmud leg.; CH, ASSAM 59114.

Calamus inermis T. Anders.

Figure 4C, D

Diagnostic characters. Stems clustered, climbing, 8-15 

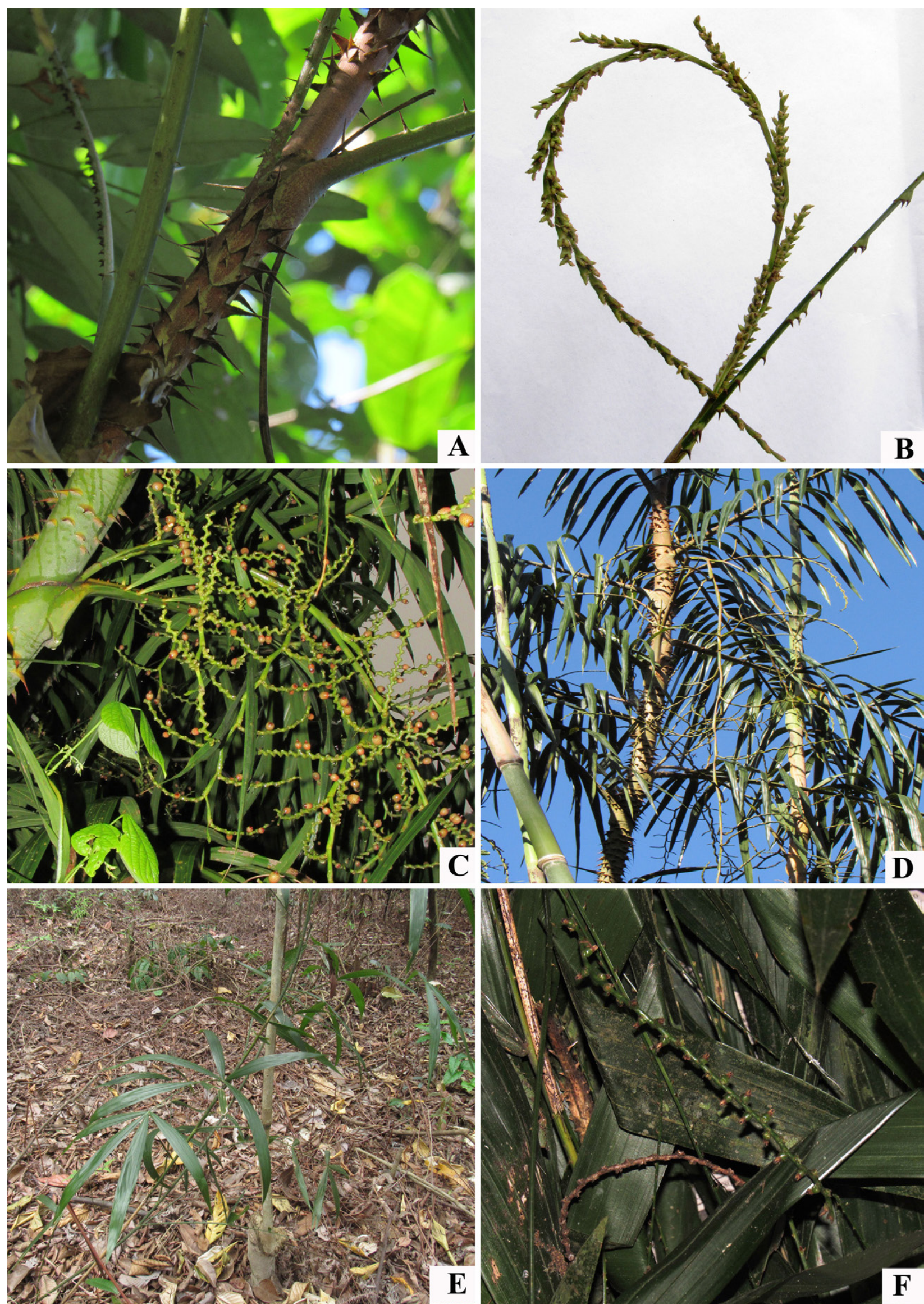

Figure 4. Palms (Arecaceae) in Assam, India. A, B. Calamus henryanus: (A) stem; (B) pistillate partial inflorescence. C, D. Calamus inermis: (C) stem with fruiting; (D) stem with staminate inflorescence. E, F. Calamus kingianus: (E) habit; (F) pistillate partial inflorescence. 
$\mathrm{m}$ long, with leaf sheath $\mathrm{ca} 10 \mathrm{~cm}$ in diameter; spines pointing downward, 1-3 cm long; indumentums brown. Leaf pinnate, 2-4 m long, cirri 1-2 $\mathrm{m}$ long; petiole 6-7 $\mathrm{cm}$ long with $4-5 \mathrm{~mm}$ long spines; leaflets many, 50-63 $\mathrm{cm}$ long in groups of $2-4$, alternate to opposite, inequidistant, lanceolate, serrated, margin bristly, acuminate, with 3 veins. Knee $6-8 \mathrm{~cm}$ long; ocrea inconspicuous, acute, ca $4 \mathrm{~mm}$ long. Staminate inflorescence ca $1.8 \mathrm{~m}$ long, terminating by ca $3-5 \mathrm{~cm}$ long non flagellate organ; partial inflorescences $32-58 \mathrm{~cm}$ long; rachillae alternate, 4-7 cm long. Pistillate inflorescence ca $1.3 \mathrm{~m}$ long; partial inflorescences ca $40 \mathrm{~cm}$ long; rachillae zigzag, 8-12 $\mathrm{cm}$ long, dyad alternate. Fruits ca $2.6 \mathrm{~cm}$ long, perianth tubular and scales in 18 vertical rows.

Flowering and fruiting. November to June.

Distribution. Dima Hasao and East Karbi Anglong districts; prefers hilly areas.

Uses. In furniture (cane).

Material examined. VIETNAM - Khanh Hoa Province, Khanh Vinh, Khanh Trung Commune; $12^{\circ} 22^{\prime} \mathrm{N}$, $108^{\circ} 49^{\prime} \mathrm{E}$; alt. $450 \mathrm{~m}$; 11 Oct. 2016; Andrew Henderson \& Nguyen Quoc Dung leg.; NYBG 02710739. INDIA • 1 \%; Assam, Dima Hasao, Jatinga; $25^{\circ} 07^{\prime} 56.3^{\prime \prime} \mathrm{N}$, 09301'15.4"E; alt. 684 m; 27 Jun. 2019; S. Mehmud leg.; CH, ASSAM 96225.

\section{Calamus kingianus Becc.}

Figure 4E, F

Diagnostic characters. Stems clustered, climbing, ca $9 \mathrm{~m}$ long; with sheath ca $1 \mathrm{~cm}$ in diameter; spines horizontal, linear ca 5-6 mm long; indumentums whitesilvery. Flagella $0.7 \mathrm{~m}$ long, spines $5-6 \mathrm{~mm}$ long. Leaf ecirriate, ca $55 \mathrm{~cm}$ long; petiole $11-12 \mathrm{~cm}$ long; leaflets 4-6 per side, single or in groups, lanceolate, ensiform, acuminate, ca $25 \mathrm{~cm}$ long, $2-2.5 \mathrm{~cm}$ wide, veins 3 , and terminally free. Knee ca $1 \mathrm{~cm}$ long, spiny; ocrea inconspicuous, ca 1-2 mm long, glabrous or spiny. Pistillate inflorescence flagellate, ca $1.1 \mathrm{~m}$ long; partial inflorescences $2-3$ in number, with single rachilla, $6-12 \mathrm{~cm}$ long.

Flowering (pistillate). March.

Distribution. Lakhimpur district.

Vernacular names. Suli Bet (Assamese).

Uses. As binding material (cane).

Material examined. INDIA - Assam; 15000 ft; Feb 1893; Dr King's collectors, leg.; CAL0000024740. • 1우 Assam, Lakhimpur, Kakoi Reserve; 27²3'46.7"N; 09405'38.1"E; alt. 118 m; 09 Mar. 2019; S. Mehmud leg.; ASSAM 96226.

\section{Calamus leptospadix Griff.}

Figure 5A, B

Diagnostic characters. Stems clustered, climbing, ca 9 $\mathrm{m}$ long, with leaf sheath ca $1.5 \mathrm{~cm}$ in diameter; spines linear ca $1-1.5 \mathrm{~cm}$ long; indumentums brown to grey; flagella $2 \mathrm{~m}$ long. Leaf ecirriate, ca $1.3 \mathrm{~m}$ long; petiole $30-40 \mathrm{~cm}$ long, with few linear spines, ca $2.5 \mathrm{~cm}$ long. Leaflets regular, 33-56 per side, alternate to opposite,
8-22 cm long, ca $1 \mathrm{~cm}$ wide, linear, serrated, acuminate, veins 3, and terminal leaflets free. Knee $2 \mathrm{~cm}$ long, smooth or spiny; ocrea divided into two parts ca $1.5 \mathrm{~cm}$ long, bristly. Inflorescence 2-4 m long, flagellate; rachillae covered by a tubular bracteole up to mid portion. Staminate partial inflorescences $12-36 \mathrm{~cm}$ long; rachillae alternate, scorpoid, 2-3 cm long; pistillate partial inflorescences 3-4 in number, $11-15 \mathrm{~cm}$ long, rachillae straight, $2-3 \mathrm{~cm}$ long, flowers dyad. Fruit globose, perianth explanate.

Flowering and fruiting. January to May and July to $\mathrm{Au}-$ gust, respectively.

Distribution. Bishwanath Chariali, Cachar, Dima Hasao, Hailakandi, West Karbi Anglong, Kokrajhar, Lakhimpur, and Tinsukia districts; prefer dry undisturbed areas.

Vernacular names. Lejai Bet (Assamese).

Uses. As vegetable (apical branch), binding material (cane).

Material examined. INDIA - Arunachal Pradesh, Dibang Valley, Dambuk; alt. 420 m; 04 Mar. 2004; M. Bhaumik leg.; ASSAM 64972. 1 \%; Assam, West Karbi Anglong, Amsoi to Amkachi Road; $26^{\circ} 02^{\prime} 51.7^{\prime \prime} \mathrm{N}, 092^{\circ}$ 25'20.6"E; alt. 423 m; 24 Feb. 2018; S. Mehmud leg.; $\mathrm{CH}$, ASSAM 96227. 1 क; Assam, Tinsukia, Jagun; $27^{\circ}$ 24'41"N, 095 56'16"E; 13 May 2019; S. Mehmud leg.; CH, ASSAM 96228.

\section{Calamus meghalayensis A. Henderson}

Figure 5C, D

Diagnostic characters. Stems clustered, climbing, ca $5 \mathrm{~m}$ long, with leaf sheath $0.8-1 \mathrm{~cm}$ in diameter; indumentums white; spines horizontal, linear 4-5 mm long; flagella ca $80 \mathrm{~cm}$ long. Knee ca $1.4 \mathrm{~cm}$ long; ocrea inconspicuous, ca $5 \mathrm{~mm}$ long, bristly. Leaf pinnate, ecirriate, ca $50 \mathrm{~cm}$ long; leaflets $4-5$ per side, in groups, lanceolate, ensiform, $20-25 \mathrm{~cm}$ long, $2-3 \mathrm{~cm}$ wide, serrated, acute, terminal leaflets connate, rarely free. Inflorescences ca $1.2 \mathrm{~m}$ long, flagella ca $25 \mathrm{~cm}$ long; bracts mouth bristly; staminate partial inflorescences $3-4$ in number, $4-10 \mathrm{~cm}$ long; rachillae alternate, ca $1 \mathrm{~cm}$ long, scorpoid; pistillate partial inflorescences $8-10 \mathrm{~cm}$ long, rachillae alternate, straight, $1-4 \mathrm{~cm}$ long with dyad flowers. Fruits globose or ellipsoid, ca $1.6 \mathrm{~cm}$ long, perianth explanate.

Flowering and fruiting. August to March and January, respectively.

Distribution. Karmup Rural district; near streams.

Vernacular names. Chorebukla (Rabha); Nari Bet (Rabha, Assamese).

Uses. As binding material (cane).

Material examined. INDIA - Assam, Kamrup, Jara Reserve; 24 May 1957; G. Panigrahi leg.; CAL. • 1

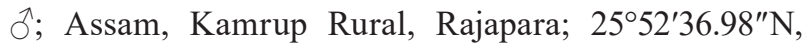
091²6'26.35"E; 08 Sep. 2019; S. Mehmud leg.; CH, ASSAM 96229. • 1우 ; same collection data as per preceding; CH, ASSAM 96230. 

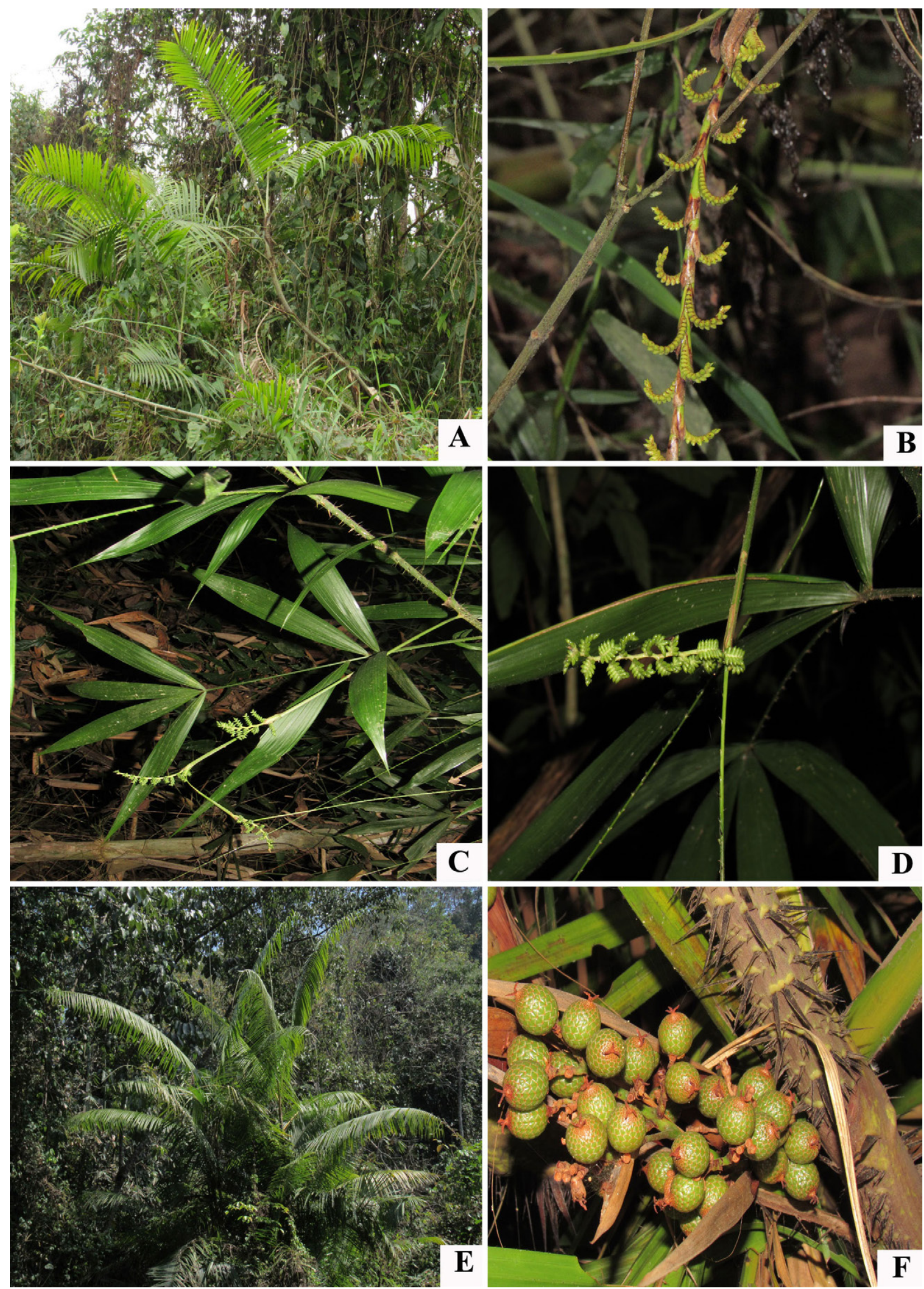

Figure 5. Palms (Arecaceae) in Assam, India. A, B. Calamus leptospadix: (A) habit; (B) partial staminate inflorescence. C, D. Calamus meghalayensis: (C) habit and pistillate inflorescence; (D) partial staminate inflorescence. E, F. Calamus melanochaetes: (E) habit; (F) fruiting. 
Calamus melanochaetes (Blume) Miquel.

Figure 5E, F

Diagnostic characters. Stems clustered, climbing, ca $15 \mathrm{~m}$ long and with leaf sheath $2.5-6 \mathrm{~cm}$ in diameter; indumentums grey; spines flat, linear, $1.5-2.2 \mathrm{~cm}$ long; flagella absent. Knee ca $8 \mathrm{~cm}$ long, spiny; ocrea inconspicuous, acute, ca $3 \mathrm{~mm}$ long. Leaf pinnate, $1-1.5 \mathrm{~m}$ long, cirri ca $1 \mathrm{~m}$ long; leaflets regular, linear, acuminate, $22-41 \mathrm{~cm}$ long, $2-3 \mathrm{~cm}$ wide, and veins 3 . Inflorescences ca $50 \mathrm{~cm}$ long; prophyll ca $45 \mathrm{~cm}$ long, boat shaped, adaxially spiny; partial inflorescence 5 or 6 in number, 10-14 cm long, covered by respective bracts similar to prophyll but glabrous; pistillate rachillae with dyad flowers; staminate flowers alternate. Fruit brown, globose, ca $1.7 \mathrm{~cm}$ in diameter, scales in 18 vertical rows. Flowering and fruiting. March to December.

Distribution. Common in Assam; prefers waterlogged plains.

Vernacular names. Houka Bet (Assamese); Gola Bet (Bengali).

Uses. In furniture (cane), Rabha community believes as lactogenic (apical stem), masticator (fruit).

Material examined. INDIA • Assam, Nawgong, Kholahat Reserve Forest; Upendranath Kanjilal leg.; ASSAM 30982. • Assam, Goalpara, Guma Reserve Forest; 21 May 1915; Upendranath Kanjilal leg.; ASSAM 30978. • Assam, Karimganj, Damchera; 2458'37.2"N, 092 $59^{\circ} 17.1^{\prime \prime} \mathrm{E}$; alt. 47 m; 18 Nov. 2018; S. Mehmud leg.; CH, ASSAM 96237.

\section{Calamus nambariensis Becc.}

Figure 6A

Diagnostic characters. Stems solitary or clustered (2 or 3 in number), ca $15 \mathrm{~m}$ long and with leaf sheath $3-4 \mathrm{~cm}$ in diameter; leaf sheath spines ca $2-3 \mathrm{~cm}$ long, pointing downwards. Flagella absent. Leaf pinnate, 2-3.5 m long, cirri ca $1.2 \mathrm{~m}$ long. Leaflets regular, lanceolate, alternate, 20-27 per side, ca $35 \mathrm{~cm}$ long and ca $2.5 \mathrm{~cm}$ wide, acute, serrated, and veins 3 . Knees ca $6 \mathrm{~cm}$ long, spiny; ocrea inconspicuous, acute 1-2 mm long. Inflorescences same as Calamus inermis.

Calamus namabariensis has been considered to be a synonym of $C$. inermis (Henderson 2020), but we recognize both the taxa as separate species by their differing number of vertical rows of fruit scales. In C. namabariensis there are 21 rows (Beccari 1908; Basu 1992; Renuka and Sreekumar 2012; Deka et al. 2018), while in C. inermis there are 18 rows (Beccari 1908; Basu 1992; Renuka et. al. 2010).

Flowering. March to June.

Distribution. Jorhat and East Karbi Anglong districts; prefers dense forest.

Vernacular names. Hauka Bet (Assamese).

Uses. In furniture (cane).

Material examined. INDIA - Assam, Nambor Wildlife Sanctuary; 20 Feb. 2016; Kishor Deka leg.; GUBH 18407. - Assam, East Karbi Anglong, Langsoipee Gaon, Koilamati Area; $26^{\circ} 21^{\prime} 21.2^{\prime \prime} \mathrm{N}, 093^{\circ} 50^{\prime 4} 49.3^{\prime \prime} \mathrm{E}$; alt. $71 \mathrm{~m}$;
20 Jun. 2019; S. Mehmud leg.; CH, ASSAM 96231.

\section{Calamus palustris Griff.}

Figure 6B

Diagnostic characters. Stems clustered, climbing, 15$20 \mathrm{~m}$ long and with leaf sheath $2-4 \mathrm{~cm}$ in diameter; flagella absent; spines $2-3 \mathrm{~cm}$ long, triangular. Knee green, ca $4 \mathrm{~cm}$ long, smooth to wrinkle; ocrea inconspicuous, acute, 5-6 $\mathrm{mm}$ long. Leaf pinnate, 3-4 $\mathrm{m}$ long, cirri 1-1.2 m long. Leaflets lanceolate, ensiform, serrated, acuminate, $19-30 \mathrm{~cm}$ long and 5-8 cm wide. Staminate inflorescences 1.2-1.5 m long; partial inflorescences 7-9 in number, ca $50 \mathrm{~cm}$ long; rachillae alternate $1.5-5 \mathrm{~cm}$ long. Pistillate inflorescence $70-80 \mathrm{~cm}$ long; partial inflorescences 5-6 in number, $18-30 \mathrm{~cm}$ long; rachillae $8-10 \mathrm{~cm}$ long.

Flowering. February to May.

Distribution. Cachar, Dhemaji, Kamrup Rural, and Kokrajhar districts; prefers waterlogged plains.

Uses. For preparation of furniture (cane).

Material examined. INDIA • North Andaman; 29 Mar. 1977; N. P. Balakrishnan leg.; CAL0000056703. • Assam,

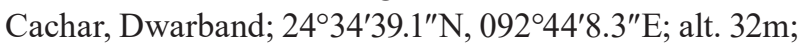
20 Nov. 2018; S. Mehmud leg.; CH, ASSAM 96232.

\section{Calamus tenuis Roxb.}

Figure 6C

Diagnostic characters. Stems clustered, climbing, 9-20 $\mathrm{m}$ long and with leaf sheath $\mathrm{ca} 2 \mathrm{~cm}$ in diameter; leaf sheath green, spines 1.5-2.5 cm long; indumentums white; flagella 1-1.1 m long. Knee 2.8-3 cm long, spiny; ocrea 5-6 mm long, unarmed. Leaf pinnate, ecirriate, 1-1.2 m long; leaflets regular, linear-lanceolate, serrated, acuminate, 30-34 in number, 29-31 cm long, 2-2.6 cm wide, equidistant, veins 3 . Staminate inflorescence $2.5-3$ $\mathrm{m}$ long, flagellate; partial inflorescences 6-9 in number, 10-22 cm long; rachillae alternate, $3-5 \mathrm{~cm}$ long. Pistillate inflorescence $2.5 \mathrm{~m}$ long; partial inflorescences 10 $13 \mathrm{~cm}$ long; rachillae alternate, $2-3 \mathrm{~cm}$ long with dyad flowers. Fruit ovate to spherical, ca $1.1 \mathrm{~cm}$ long, perianth tubular, scales in 15 vertical rows.

Flowering and fruiting. July to September and December to March, respectively.

Distribution. Throughout Assam; prefers waterlogged plains.

Vernacular names. Jati Bet, Pani Bet (Assamese).

Uses. For furniture (cane), masticator (fruit) food (apical stem).

Material examined. INDIA • 1 त; Assam, North Lakhimpur; Jan. 1940; no. 19010; ASSAM 30943. - Assam,

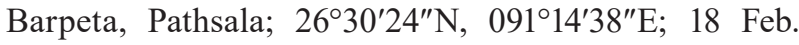
2018; S. Mehmud leg.; CH, ASSAM 96233.

\section{Key to the species of Caryota}

1a Solitary; leaflets apex not elongated Caryota obtusa 

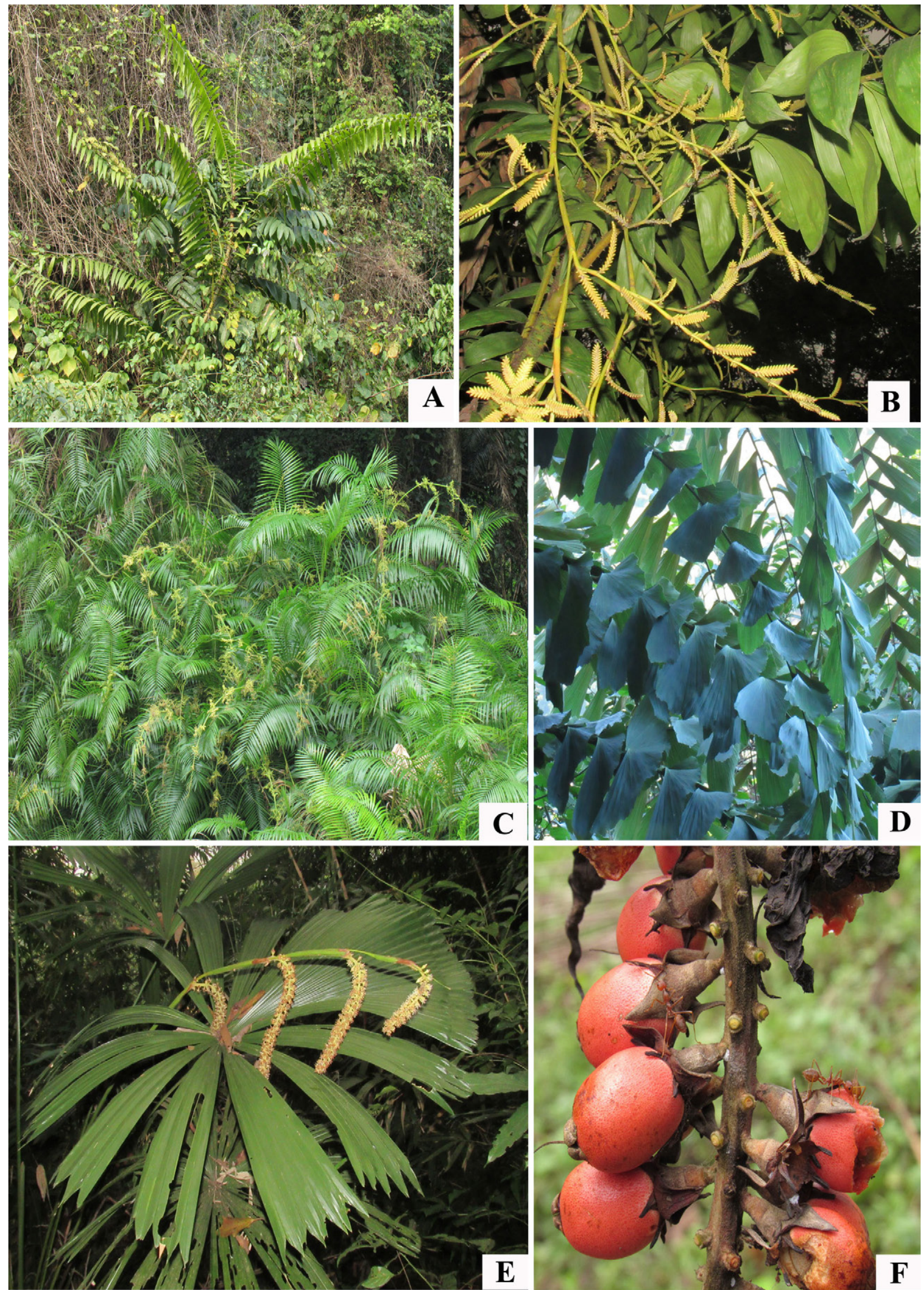

Figure 6. Palms (Arecaceae) in Assam, India. A. Calamus nambariensis, habit. B. Calamus palustris, stem and leaf with staminate inflorescence. C. Calamus tenuis, habit with staminate inflorescence. D. Caryota obtusa leaflets. E, F. Licuala peltata: (E) habit; (F) fruiting. 
1b Clustered or solitary; leaflets apex elongated Caryota urens

\section{Caryota obtusa Griff.}

Figure 6D

Diagnostic characters. Solitary, erect, $20-35 \mathrm{~m}$ tall woody palm tree; trunk $70-80 \mathrm{~cm}$ in diameter. Leaf bipinnate, 7-8 $\mathrm{m}$ long, at the top, spreading; rachis greenbrown; primary pinnae alternate, gradually short towards apex; secondary pinnae $18-22$ in number per side, jagged apex not elongated.

Distribution. Dima Hasao district; prefers sun exposed hilly areas.

Material examined. INDIA • NEFA (North-East Frontier Agency), Lohit; 17 Dec. 1969; J. Joseph leg.; ASSAM 53777. • Assam, Dima Hasao, Jatinga Hill; $25^{\circ} 06^{\prime} 41^{\prime \prime} \mathrm{N}$, 09302'32"E; alt. 898 m; 14 Nov. 2019; S. Mehmud leg.; CH, ASSAM 96234.

\section{Caryota urens $\mathbf{L}$.}

Diagnostic characters. Clustered or solitary, 20-25 m tall, woody palm tree; internodes $30-35 \mathrm{~cm}$ long and 30 $50 \mathrm{~cm}$ in diameter. Leaf bipinnate, 5-7 m long; sheath green, 2-3 m long, margin with black fibre. Primary pinnae 10-16 in number, gradually short towards apex; secondary pinnae alternate, linear, 25-34 cm long, jagged and tip elongated. Inflorescences below the leaf, 2-3 m long; rachillae 1-1.2 m long; pistillate flower globose, $0.8-1.1 \mathrm{~cm}$ long, in between two ovate staminate flowers ca $3 \mathrm{~cm}$. Fruit red to purple, round, $1.5 \mathrm{~cm}$ in diameter.

Flowering and fruiting. June to January and September to November, respectively.

Distribution. Throughout Assam; on plains and in hilly areas.

Vernacular names. Sau (Assamese).

Uses. As masticator (fruit), handle sticks and in handlooms (mature stem).

Material examined. INDIA • Assam, Barnadi WLS, Bogamati; alt. 190 m; 12 Sep. 2011; S. R. Talukdar leg.; ASSAM 84162. • Assam, Jorhat, Gibbon WLS; 16 Jun. 2010; Ranjit Daimari leg.; ASSAM 81028. • Assam, Dhemaji, Pera Bhari; $27^{\circ} 33^{\prime} 27.4^{\prime \prime} \mathrm{N}, 094^{\circ} 35^{\prime} 18.9^{\prime \prime} \mathrm{E}$; alt. $53 \mathrm{~m}$; 08 May 2019; S. Mehmud leg.; CH, ASSAM 96235.

\section{Cocos nucifera $\mathrm{L}$.}

Diagnostic characters. Solitary, erect, $15-20 \mathrm{~m}$ tall woody palm tree; trunk brown, $30-35 \mathrm{~cm}$ in diameter. Leaf pinnate, $4-6 \mathrm{~m}$ long; petiole green, brown fibre at base; leaflets regular, $120-130$ per side, $0.7-1 \mathrm{~m}$ long, linear, entire, acuminate with prominent midvein. Inflorescences $0.6-1 \mathrm{~m}$ long; bracts brown; pistillate flowers at base, staminate flowers at apex of the rachillae; fruits ovate, brown ca. $15 \mathrm{~cm}$ in diameter.

Flowering and fruiting. Throughout the year.

Distribution. Throughout Assam.

Vernacular names. Narikol (Assamese).
Uses. Food, oil (fruit), firewood (leaf, stem), broom (midvein).

Material examined. INDIA - Assam, Marigaon; $26^{\circ} 12^{\prime}$ $30^{\prime \prime N}$, 092 $03^{\prime} 12^{\prime \prime}$; alt. 1 m; 08 Apr. 2018; S. Mehmud leg.; $\mathrm{CH}$.

\section{Corypha umbraculifera $\mathrm{L}$.}

Diagnostic characters. Solitary erect, $15-18 \mathrm{~m}$ tall woody palm tree, hapaxanthic, $60-70 \mathrm{~cm}$ in diameter; trunk black, irregular in size. Leaf costapalmate, 5-5.5 $\mathrm{m}$ long; petiole 3-3.5 m long, margins with $2 \mathrm{~cm}$ long sharp black spines; leaflets $2.4-3 \mathrm{~m}$ long, apices bifid, ca $10 \mathrm{~cm}$ long. Inflorescence terminal, ca $2 \mathrm{~m}$ long, spreading in all directions. Fruits round, black to violet, ca 1.8 $\mathrm{cm}$ in diameter, solitary or in pairs.

Flowering and fruiting. March to August.

Distribution. Kamrup Metro.

Uses. Ornamental.

Material examined. INDIA - Calcutta; 23 Jul. 1898; M. Device leg.; CAL 495172. • Assam, Kamrup Metro, Near Dighali Pukhuri; $26^{\circ} 11^{\prime} 18.6^{\prime \prime} \mathrm{N}, 091^{\circ} 45^{\prime} 17.8^{\prime \prime} \mathrm{E}$; alt. $14 \mathrm{~m}$; 30 Mar. 2018; S. Mehmud leg.; CH, ASSAM 96236.

\section{Key to the species of Dypsis}

1a Stems solitary, ca $25 \mathrm{~cm}$ in diameter; leaf arranged in three directions; indumentums brown

Dypsis decaryi

1b Stems clustered, ca $9-10 \mathrm{~cm}$ in diameter; leaf not arranged in three directions; indumentums silvery

Dypsis lutescens

\section{Dypsis decaryi (Jum.) Beenjte \& J. Dransf.}

Diagnostic characters. Stem solitary, erect, blackbrown, unarmed, $2.5-4 \mathrm{~m}$ tall and $30-32 \mathrm{~cm}$ in diameter. Leaf pinnate, ca $2.5 \mathrm{~m}$ long; petiole with red and black indumentums; leaves arranged in three directions; leaflets regular, linear, 54-67 cm long, apices bifid. Inflorescence branched and below the leaf, $50-60 \mathrm{~cm}$ long.

Flowering. October to November

Distribution. Throughout Assam.

Uses. Ornamental.

Material examined. USA - California, San Diego; $32^{\circ} 44^{\prime} \mathrm{N}, 117^{\circ} 10^{\prime} \mathrm{W}$; alt. $30 \mathrm{~m}$; 9 Nov. 1995 ; Jay B. Walker leg.; NYBG 00201925. INDIA • Assam, Kamrup Metro, Guwahati; $26^{\circ} 08^{\prime} 34^{\prime \prime} \mathrm{N}, 091^{\circ} 47^{\prime} 11^{\prime \prime} \mathrm{E}$; alt. $67 \mathrm{~m}$; $13 \mathrm{Jul}$. 2018; S. Mehmud leg.; CH, ASSAM 96238.

\section{Dypsis lutescens (H. Wendle.) Beentje \& J. Dransf.}

Diagnostic characters. Stems clustered, erect, 4-6 m tall; internodes green, $8-10 \mathrm{~cm}$ long and $5-7 \mathrm{~cm}$ in diameter. Leaf pinnate, $2-2.5 \mathrm{~m}$ long; crownshaft $40-46 \mathrm{~cm}$ long, indumentums silvery white; petiole green, 20-45 $\mathrm{cm}$ long. Leaflets regular, $45-61 \mathrm{~cm}$ long, ca $2 \mathrm{~cm}$ wide, opposite, linear, glabous, ensiform, entire, apices bifid, acute. Inflorescence branched, 0.9-1.1 m long, below the crownshaft; rachillae 9-10 cm long; pistillate flower in 
between two staminate flowers. Fruits ellipsoid, $1 \mathrm{~cm}$ long, yellow.

Flowering and fruiting. January and June to August, respectively.

Distribution. Throughout Assam.

Vernacular names. Momai Tamul (Assamese).

Uses. Ornamental; as masticator (fruit).

Material examined. WEST INDIES - Tobago; 8 Apr. 1913; W. E. Broadway leg.; NYBG 1662698. INDIA - Assam, Kamrup Metro, Panbazar; 26¹1'10"N,

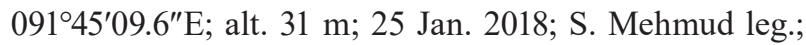
CH, ASSAM 96239.

\section{Elaeis guineensis Jacq.}

Diagnostic characters. Stems solitary, erect, 10-15 m tall; trunk with old petioles; leaf pinnate, 3-4 m long; petiole margin spiny, $1.5-2 \mathrm{~cm}$ long; leaflets, linear, opposite, 50-68 $\mathrm{cm}$ long, ca $3 \mathrm{~cm}$ wide, with prominent midvein. Staminate inflorescence interfoliar, $20-23 \mathrm{~cm}$ long; rachillae elongated, $15-18 \mathrm{~cm}$ long and terminated by a hook like tip, ca $1 \mathrm{~cm}$ long. Pistillate inflorescence ca $15 \mathrm{~cm}$ long, globose; partial inflorescences $9-11 \mathrm{~cm}$ long. Fruits ellipsoid, black, $3 \mathrm{~cm}$ long.

Flowering and fruiting. September to November.

Distribution. Cachar, Goalpara, Kamrup Rural, and Marigaon districts.

Vernacular names. Oil palm (English).

Uses. Palm oil (nut).

Material examined. VIETNAM - Phu Tho Province, Phu Ninh, Tram Than mun; $21^{\circ} 29.21^{\prime} \mathrm{N}, 105^{\circ} 13.33^{\prime} \mathrm{E}$; alt. 33 m; 06 Jun. 2006; Phan Ke Loc, Nguyen Sinh Khang, $\mathrm{Vu}$ Xuan Manh, Pham Van The, To Van Thao, leg.; NYBG 02384543. INDIA - Assam, Marigaon, Nelie; $26^{\circ} 41^{\prime} 10.9^{\prime \prime} \mathrm{N}, 094^{\circ} 21^{\prime} 12.6^{\prime \prime} \mathrm{E}$; alt. 43 m; 16 Sep. 2018; S. Mehmud leg.; CH, ASSAM 96240.

\section{Licuala peltata Roxb. ex Buch.-Ham.}

Figure 6E, F

Diagnostic characters. Stems solitary, 2-4 $\mathrm{m}$ tall and ca $10 \mathrm{~cm}$ in diameter; trunk with old petioles. Leaf palmate, orbicular, ca $2 \mathrm{~m}$ long; leaflets $15-22$ in number, folded, apices bifid, acute; petiole $1.5 \mathrm{~m}$ long, margin with spines, 2-3 cm long. Inflorescence 3-3.5 m long, rachis round; partial inflorescences $4-5$ in number, unbranched, 42-65 cm long; bracts tubular. Flowers bisexual, ca $2 \mathrm{~cm}$ long, creamy yellowish. Fruits round to ellipsoid, red, ca $2.5 \mathrm{~cm}$ long.

Flowering and fruiting. September to January and July to August, respectively.

Distribution. Cachar, Hailakandi, Goalpara, Golaghat, Karimganj and Kamrup Rural districts; observed in hills and open fields.

Vernacular names. Japi Goch (Rabha).

Uses. Ornamental; as masticator (fruit), traditional "Japi" hat (leaf).

Material examined. INDIA • Assam, Cachar, Borail
Wildlife Sanctuary; 26 Apr. 2011; Hussain Ahmed Borbhuiyan leg.; ASSAM 84947. - Assam, Kamrup Rural, Chandubi; 2552'36"N, 091 26'18"E; 14 Sep. 2017; S. Mehmud leg.; CH, ASSAM 96241.

\section{Key to the species of Livistona}

1a Leaflets apices dropping; fruits ellipsoid, bluishgreen Livistona chinensis

1b Leaflets apices not dropping; fruits round, bluishblack or red 2

2a Stem ca 20 m tall; fruit red

2b Stem 12-15 m tall; fruit bluish-black Livistona jenkinsiana

\section{Livistona chinensis (Jacq.) R.Br. ex Mart.}

Diagnostic characters. Stems solitary, erect, 6-9 m tall and $20-30 \mathrm{~cm}$ in diameter, grey. Leaf costapalmate, 3-4 $\mathrm{m}$ long; petiole 2-2.5 m long, black fibre at base, margin spiny, ca $2 \mathrm{~cm}$ long; lamina ca $1.3 \mathrm{~m}$ long. Leaflets 90-100 in number, ca $60 \mathrm{~cm}$ long, apices bifid, ca $30 \mathrm{~cm}$ long, acuminate and dropping. Inflorescence $1.3-1.5 \mathrm{~m}$ long; primary branches 6-7 in number, $30-45 \mathrm{~cm}$ long; bracts tubular, $20-25 \mathrm{~cm}$ long, indumentums brown. Fruits ellipsoid, bluish-green, 2-2.5 cm long.

Flowering and fruiting. March to April and September to October, respectively.

Distribution. Throughout Assam.

Uses. Ornamental.

Material examined. NETHERLANDS ANTILLES • Saba; $17^{\circ} 37^{\prime} 37^{\prime \prime} \mathrm{N}, 063^{\circ} 13^{\prime} 54^{\prime \prime} \mathrm{W}$; alt. $380 \mathrm{~m}$; 11 Mar. 2007; S. A. Mori, C. A. Gracie, W. R. Buck, H. Sipman \& S. Nielsen leg.; NYBG 00933709. INDIA • Assam, Kamrup Rural, Rani; $26^{\circ} 02^{\prime} 49^{\prime \prime} \mathrm{N}, 091^{\circ} 35^{\prime} 21^{\prime \prime} \mathrm{E}$; alt. 53 m; 11 Nov. 2017; S. Mehmud leg.; CH, ASSAM 96242.

\section{Livistona jenkinsiana Griff.}

Figure 7A, B

Diagnostic characters. Stems solitary, erect, 10-15 m tall tree, 25-30 cm in diameter; trunk grey-black, with old petioles. Leaf costapalmate, 2.2-3 m long; petiole $1.5-2 \mathrm{~m}$ long, margin with brown curved spines, ca 1.5 $\mathrm{cm}$; lamina $0.9-1.2 \mathrm{~m}$; leaflets $46-53$ in number, apices bifid, 6-7 cm long, acute. Inflorescence 1.3-1.4 m long; 3 branched, 70-87 cm long; rachillae 10-42 cm long. Fruits round, bluish-black, ca $2.4 \mathrm{~cm}$ in diameter.

Flowering and fruiting. February and May to June, respectively.

Distribution. Baksa, Darrang, Dhemaji, Dibrugarh, Jorhat, Kamrup Rural, Lakhimpur, Nagaon, Sibsagar and Tinsukia districts; wild and domesticated.

Vernacular names. Takou (Assamese).

Uses. For brooms, huts and hats (leaf), masticator (fruit). Material examined. INDIA - Assam, Kamrup Rural,

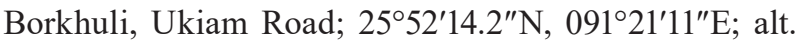
66 m; 01 Jul. 2018; S. Mehmud leg.; CH, ASSAM 96243. 


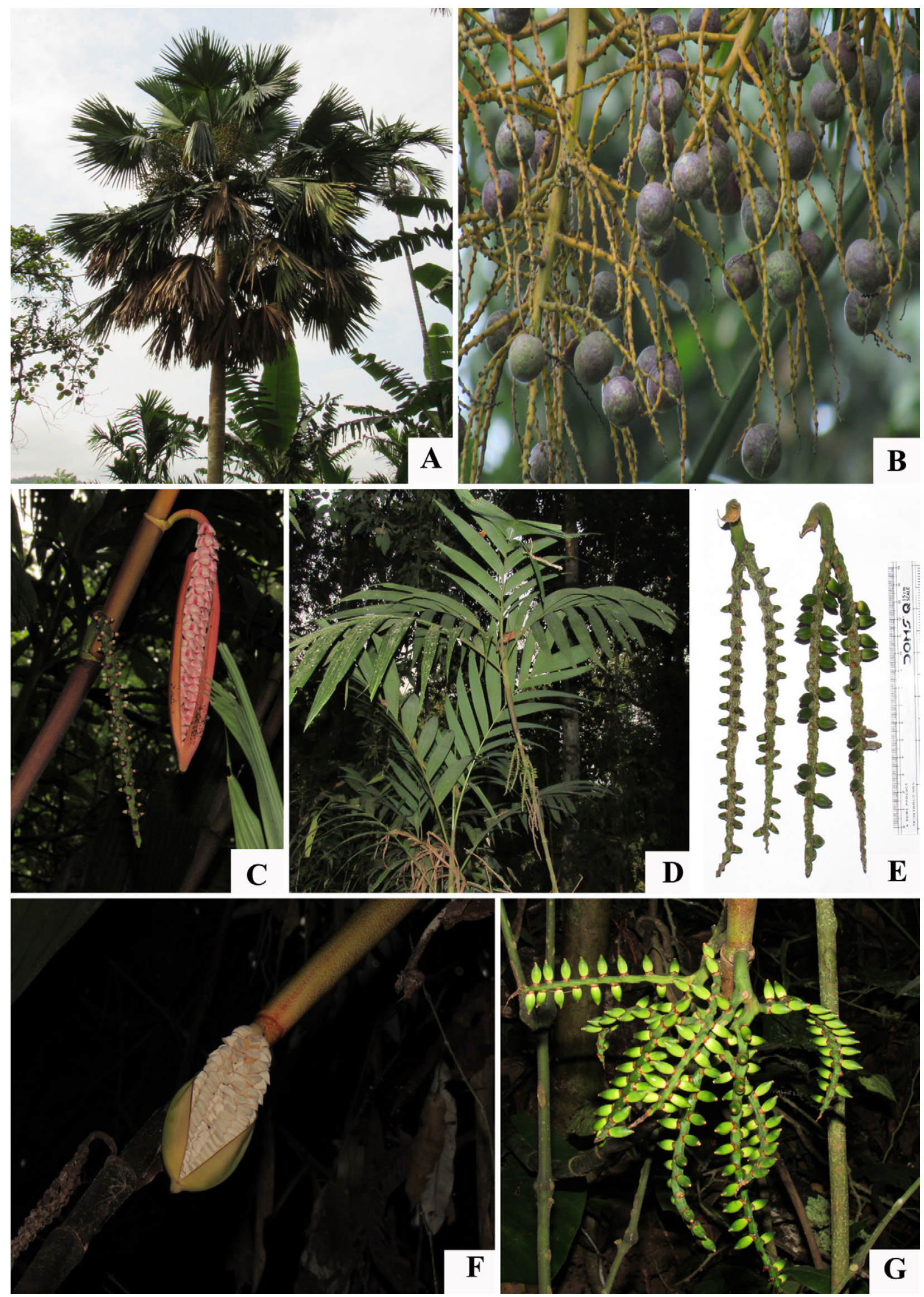

Figure 7. Palms (Arecaceae) in Assam, India. A, B. Livistona jenkinsiana: (A) habit; (B) fruit. C. Pinanga gracilis, flowering. D, E. Pinanga griffithii: (D) habit, (E) fruiting. F, G. Pinanga sylvestris: (F) flowering; (G) fruiting. 


\section{Livistona rotundifolia (Lam.) Mart.}

Diagnostic characters. Stems solitary, erect, 25-30 m tall tree, $22-30 \mathrm{~cm}$ in diameter; trunk smooth and grey. Leaf costapalmate, 1.8-2.9 m long; petiole curved at the base with brown fibre, red, margin spiny; lamina ca $1 \mathrm{~m}$ long, leaflets $0.7-1 \mathrm{~m}$ long, apices bifid, ca $12 \mathrm{~cm}$ long, acute, glabrous. Inflorescence three branched, 1.8-2 m long; each branch with 6-7 partial inflorescences, 12 $26 \mathrm{~cm}$ long; rachilla 4-7 cm long; flowers yellow. Fruits round, red, ca $1 \mathrm{~cm}$.

Flowering and fruiting. April to August.

Distribution. Throughout Assam.

Uses. Ornamental.

Material examined. USA - Hawaii, University of Hawaii Campus; 19 Feb. 1962; V. J. Krajina leg.; NYBG 23195621. INDIA - Assam, Kamrup Metro, Guwahati, Panbazar; 26¹1'10.9"N, 09145'07.4"E; alt. 72 m; 05 Apr. 2018; S. Mehmud leg.; CH, ASSAM 96244.

\section{Key to the species of Phoenix}

1a Palm ca $4 \mathrm{~m}$ tall; leaflets arrange in one plane, tip not sharp Phoenix roebelenii

1b Palm ca $30 \mathrm{~m}$ tall; leaflets arrange in two planes, tip sharp Phoenix sylvestris

\section{Phoenix roebelenii O'Brien, Gard.}

Diagnostic characters. Stems solitary, 1-2 $\mathrm{m}$ tall shrubs, and ca $10 \mathrm{~cm}$ in diameter. Leaf pinnate, $1-1.5 \mathrm{~m}$ long; petiole green, 23-25 cm long; acathnophylls 1-7 $\mathrm{cm}$ long. Leaflets $40-42$ per side, linear, opposite to alternate, 14-33 cm long, glabrous, entire, acuminate, tip not sharp, and arrange in one plane. Inflorescences 35 $37 \mathrm{~cm}$ long, rachis light green, 22-25 cm long; rachillae 4-12 cm long: staminate flowers ca $1 \mathrm{~cm}$ long, sweet scented, creamy yellowish; pistillate flowers 3-4 mm long. Fruits obovoid to ellipsoid, purplish-brown, ca 1 cm long.

Flowering and fruiting. April to May and July to August, respectively.

Uses. Ornamental.

Material examined. INDIA • Assam, Kamrup Metro, Panbazar; $26^{\circ} 11^{\prime} 10^{\prime \prime} \mathrm{N}, 091^{\circ} 45^{\prime} 9^{\prime \prime} \mathrm{E}$; alt. 77 m; 17 Apr. 2018; S. Mehmud leg.; CH, ASSAM 96245.

\section{Phoenix sylvestris (L.) Roxb.}

Diagnostic characters. Stems solitary, erect, 14-20 m tall tree, rarely branched; trunk with old petioles, 30$35 \mathrm{~cm}$ in diameter. Leaf pinnate, $2-3.5 \mathrm{~m}$ long; acanthophylls $10 \mathrm{~cm}$ long; leaflets $30-40 \mathrm{~cm}$ long, linear, entire, tip sharp and arrange in two planes. Staminate inflorescence erect; rachis ca $14 \mathrm{~cm}$ long; rachillae 9-13 cm long; flowers creamy white, ca $8 \mathrm{~mm}$ long. Pistillate inflorescence 50-90 cm long; rachillae 32-36 cm long; flowers green, ca $6 \mathrm{~mm}$ long. Fruits ellipsoid, yellow 2.5-3 cm long.

Flowering and fruiting. December to February and May to June, respectively.
Distribution. Throughout Assam.

Vernacular names. Khejur (Assamese).

Uses. Food (fruit), fire woods (leaf).

Material examined. INDIA • Assam, Tezpur (Darrang); 23 May 1948; G. K. Deka leg.; ASSAM 37686. • Assam, Darrang, Baihata; 26²0'49"N, 09146'59"E; alt. 27 m; 19 May 2019; S. Mehmud leg.; CH, ASSAM 96246.

\section{Key to the species of Pinanga}

1a Leaflets 3-5 in number; inflorescence unbranched; fruits spirally arranged ............. Pinanga gracilis

1b Leaflets more than 5 in number; inflorescence branched; fruits not spirally arranged 2

2a Fruit arrangement opposite ..... Pinanga sylvestris

2b Fruit arrangement in three directions

Pinanag griffithii

\section{Pinanga gracilis $\mathrm{BI}$.}

Figure 7C

Diagnostic characters. Stems clustered, erect, brownblack, unarmed, 3-4 m tall; internodes 7-10 cm long and ca $1.3 \mathrm{~cm}$ in diameter. Leaf pinnate, $0.6-1 \mathrm{~m}$ long; crownshaft $12-16 \mathrm{~cm}$ long, with golden indumentums; petiole 6-10 cm long. Leaflets regular, opposite to subopposite, 3-5 per side, lanceolate to sigmoid, 30-33 cm long, ca 5 $\mathrm{cm}$ wide, acuminate at the base with 5 or 6 veins; terminal leaflets connate at base, ca $30 \mathrm{~cm}$ long and ca $8 \mathrm{~cm}$ wide, tip dentate and apices bifid, entire, veins 9 or 10 . Inflorescence below the leaf, $20-33 \mathrm{~cm}$ long with single rachilla; pistillate flower small, ca $5 \mathrm{~mm}$, in between two staminate pink flowers, ca $8 \mathrm{~mm}$ wide. Fruits ovoid, red, arrangement spiral, ca $1.5 \mathrm{~cm}$ long.

Flowering and fruiting. May to June and November to December, respectively.

Distribution. Cachar, Dibrugarh, Jorhat, Kamrup Metro, East Karbi Anglong, West Karbi Anglong, Kokrajhar and Tinsukia districts.

Vernacular names. Geruga Tamul (Assamese); Ram Gua (Bengali, Dimasha).

Uses. As masticator (fruit).

Material examined. INDIA - Assam, Nongpoh Forest; 14 Dec. 1931; Sri Ram leg.; ASSAM 30871. • Assam, Cachar, Daulashal; 24²6'14"N, 09249'34.6"E; alt. 5 m; 15 Nov. 2019; S. Mehmud leg.; CH, ASSAM 96247.

\section{Pinanga griffithii Becc.}

Figure 7D, E

Diagnostic characters. Stems clustered, erect, 1.5-2.5 $\mathrm{m}$ long; internodes $7-8 \mathrm{~cm}$ long and $2-2.5 \mathrm{~cm}$ in diameter. Leaf pinnate, ca $1.1 \mathrm{~m}$ long; crownshaft $25-38 \mathrm{~cm}$ long, indumentums brown; petiole $15-22 \mathrm{~cm}$ long. Leaflets $9-13$ per side, $32-43 \mathrm{~cm}$ long, $6-8 \mathrm{~cm}$ wide, alternate, lanceolate, entire, acuminate, basal leaflets with 2 or 3 veins; terminal leaflets connate, ca $30 \mathrm{~cm}$ long, 5-12 $\mathrm{cm}$ wide, veins 5 or 6 . Inflorescence $22-28 \mathrm{~cm}$ long; rachis $4-5 \mathrm{~cm}$ long; rachillae $2-4$ in number, $19-24 \mathrm{~cm}$ 
long. Fruits ellipsoid, arrangement in three directions, ca $1.7 \mathrm{~cm}$ long.

Flowering and fruiting. September and November to February, respectively.

Distribution. Cachar and Tinsukia districts; in shaded hilly areas.

Vernacular names. Geruga Tamul (Assamese).

Uses. As masticator (fruit).

Material examined. INDIA - Assam, South Lushan Hill; no. 117; CAL 492018. • Assam, Cachar, Daulashal, Inner Line Forest; 2426'29.4"N, 092 49'26"E; alt. 13 m; 15 Nov. 2018; S. Mehmud leg.; CH, ASSAM 96248.

\section{Pinanga sylvestris (Lour.) Hodel.}

Figure 7F, G

Diagnostic characters. Stem clustered, erect, 2-2.6 m tall; internodes black, ca $18 \mathrm{~cm}$ long and ca $2 \mathrm{~cm}$ in diameter. Leaf pinnate, $1.2-1.5 \mathrm{~m}$ long; crownshaft ca 30 $\mathrm{cm}$ long, indumentums black-brown; petiole $30-35 \mathrm{~cm}$ long; leaflets $11-18$ per side, opposite or alternate, linear to lanceolate, $30-52 \mathrm{~cm}$ long, $1-3.5 \mathrm{~cm}$ wide, veins 2 or 3 , terminal leaflets connate. Inflorescence ca $22 \mathrm{~cm}$ long; rachillae 2-4 in number, ca. $19 \mathrm{~cm}$ long; pistillate flower ca $5 \mathrm{~mm}$ long, in between two white staminate flowers, ca $7 \mathrm{~mm}$ wide. Fruits ovate, arrangement opposite, ca 1.4 cm long.

Fruiting and flowering. June to July.

Distribution. Cachar, Hailakandi, Jorhat, Kamrup Rural and West Karbi Anglong, districts; near streams, and shaded areas.

Vernacular names. Geruga Tamul (Assamese); Khidali (Tiwa); Koibir (Karbi).

Uses. As masticator (fruit).

Material examined. INDIA - Assam, West Karbi Anglong, Umtereng; 2550'49.7"N, 092 ${ }^{\circ} 14^{\prime} 6.6^{\prime \prime} \mathrm{E}$; alt. $736 \mathrm{~m}$; 09 Jun. 2018; S. Mehmud leg.; CH, ASSAM 96249.

\section{Plectocomia assamica Griff.}

Figure 8A, B

Diagnostic characters. Stems clustered, climbing, 12-18 $\mathrm{m}$ long and $8-15 \mathrm{~cm}$ in diameter; indumentums silverybrown; spines linear, greenish to white, $2-3 \mathrm{~cm}$ long. Flagella, knee and ocrea are absent. Leaf pinnate, ca $3.5 \mathrm{~m}$ long, cirri ca $1.5 \mathrm{~m}$ long. Leaflets up to 93 per side, regular, alternate, lanceolate, $42-52 \mathrm{~cm}$ long, 5-7.3 cm wide, abaxially white indumentums. Pistillate inflorescence 1.8-2.3 m long, flagella absent; partial inflorescence 6 or 7 in number, alternate, $1-1.2 \mathrm{~m}$ long, bract tubular; rachilla $3-4 \mathrm{~cm}$ long, alternate, covered by $5-6 \mathrm{~cm}$ long bract, tip triangular, adaxially with yellow spores; flowers scented, creamy-yellowish, 4 or 5 per side, bracteole acute, ca $4 \mathrm{~mm}$ long. Fruit round, red-brown, scales arranged in ca 53 vertical rows, margin hairy.

Flowering (pistillate) and fruiting. February to March. Distribution. Cachar, Dibrugarh and Lakhimpur districts; in hilly areas, near streams.

Vernacular names. Rai Bet (Dimasha); Raja Bet
(Assamese); Rani Bet (Rabha).

Uses. For furniture (cane).

Material examined. INDIA - Assam; 15 Dec. 1937; ASSAM 30915. - 19; Assam, Cachar, Daulashal; 242 $7^{\prime} 01.1^{\prime \prime} \mathrm{N}, 092^{\circ} 48^{\prime} 50^{\prime \prime} \mathrm{E}$; alt. 46 m; 27 Mar. 2019; S. Mehmud leg.; $\mathrm{CH}$, ASSAM 96250.

\section{Ptychosperma macarthurii (H.Wendl. ex H.J.Veitch) H.Wendl. ex Hook.f.}

Diagnostic characters. Stems clustered, erect, unarmed, 2-4 m tall; internodes $12-16 \mathrm{~cm}$ long and $4-6 \mathrm{~cm}$ in diameter. Leaf pinnate, ca $1.5 \mathrm{~m}$ long; crownshaft green with silvery wax; leaflets $28-35$ per side, linear, alternate to sub opposite, midvein adaxially prominent. Inflorescence below the leaf, 35-38 cm long, branched; rachillae $14-15 \mathrm{~cm}$ long. Fruit ovate, round, red, ca $1.5 \mathrm{~cm}$ in diameter, seed grooved.

Flowering and fruiting. January to February and $\mathrm{Au}-$ gust to September, respectively.

Uses. Ornamental.

Material examined. INDIA • Madras, Pandichery; 12 May 1961; Ph. Guinet leg.; ASSAM 495109. • Assam, Kamrup Metro, Guwahati; 261'01"N, 09146'24"E; alt. 82 m; 16 Feb. 2018; S. Mehmud leg.; CH, ASSAM 96251.

\section{Rhapis excelsa (Thunb.) Henry}

Diagnostic characters. Stems clustered, erect, unarmed, ca $1.5 \mathrm{~m}$ long and ca $1 \mathrm{~cm}$ in diameter; covered by black fibre of leaf sheath. Leaf palmate, alternate, $30-32 \mathrm{~cm}$ long; petiole ca $16 \mathrm{~cm}$ long; leaflets 6 or 7 in number, $15-$ $18 \mathrm{~cm}$ long, connate at the base up to $1-1.2 \mathrm{~cm}$, free parts oblong to linear, ensiform, and veins 2 or 3 .

Uses. Ornamental.

Material examined. USA - California, San Diego; $32^{\circ}$ $44^{\prime} \mathrm{N}, 117^{\circ} 10^{\prime} \mathrm{W}$; alt. 30 m; 16 Feb. 1995; Jay B. Walker leg.; NYBG 00201827. INDIA • Assam, Kamrup Metro, Guwahati; $26^{\circ} 11^{\prime} 10^{\prime \prime} \mathrm{N}, 091^{\circ} 45^{\prime} 09.6^{\prime \prime} \mathrm{E}$; alt. $77 \mathrm{~m}$; 14 Jun. 2018; S. Mehmud leg.; CH, ASSAM 96252.

\section{Roystonea regia (Kunth.) O. F. Cook}

Diagnostic characters. Stems solitary, erect, 20-30 m tall woody tree, and ca $50 \mathrm{~cm}$ in diameter, grey, irregular in size. Leaf pinnate; crownshaft ca $1 \mathrm{~m}$ long, green; rachis ca 2-3 m long; leaflets $42-51 \mathrm{~cm}$ long, linear. Inflorescence below the leaf, upwards, 1.2-1.4 m long, branched; spathe green, cylindrical, ca $2 \mathrm{~m}$ long. Staminate flowers ca $6 \mathrm{~mm}$, and pistillate flowers ca $3 \mathrm{~mm}$; fruit round.

Flowering and fruiting. June to July and January to February, respectively.

Uses. Ornamental.

Material examined. WEST INDIES • Apr. 1904; A. H. Carties leg.; CAL. INDIA - Assam, Kamrup Metro, Maligaon; 2609'38"N, 09142'37"E; alt. 63 m; 18 Feb. 2018; S. Mehmud leg.; $\mathrm{CH}$. 


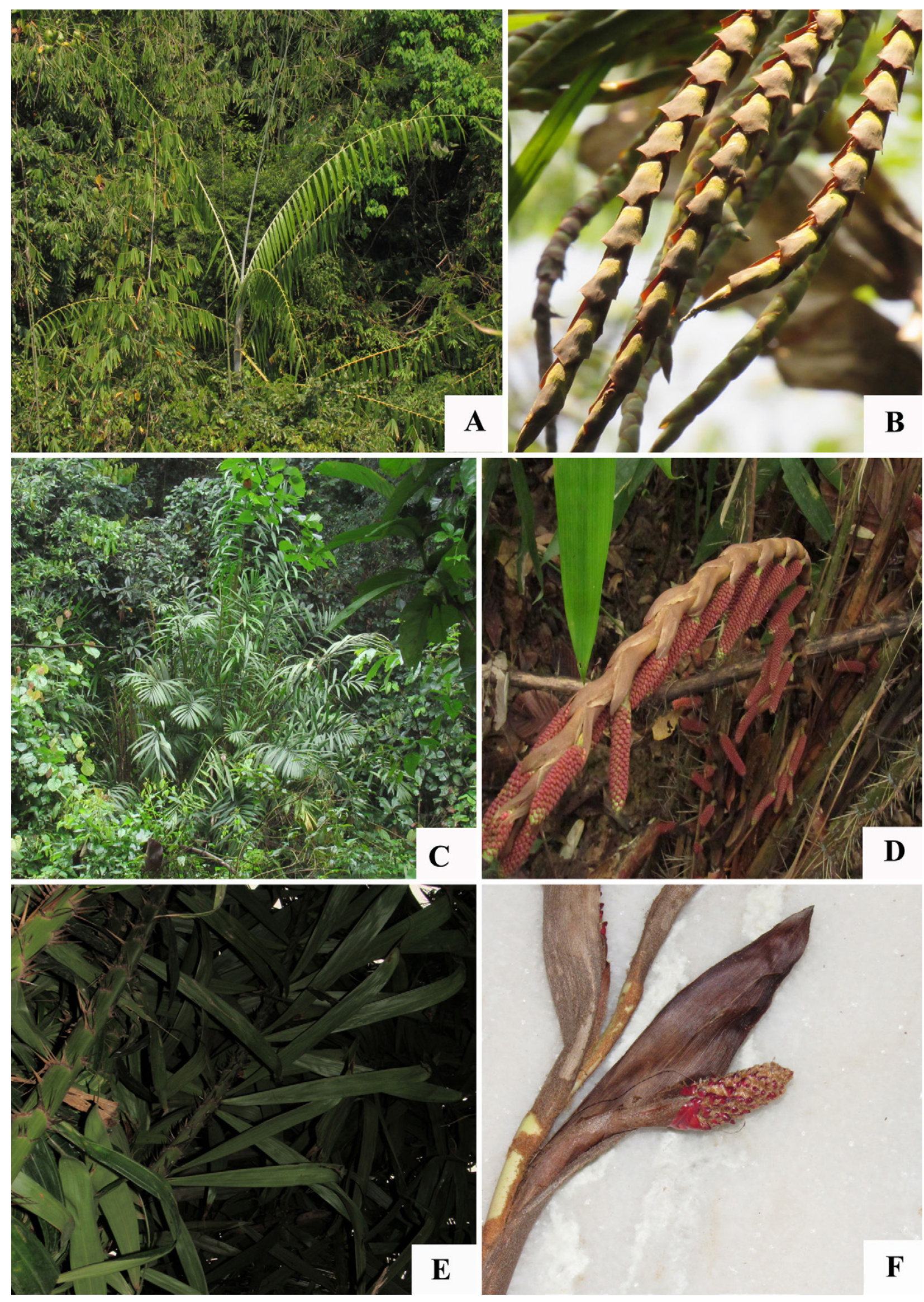

Figure 8. Palms (Arecaceae) in Assam, India. A, B. Plectocomia assamica. A. Habit. B. Pistillate inflorescence. C, D. Salacca secunda. C. Habit. D. Staminate inflorescence. E, F. Salacca wallichiana. E. Leaf. F. Inflorescence. 


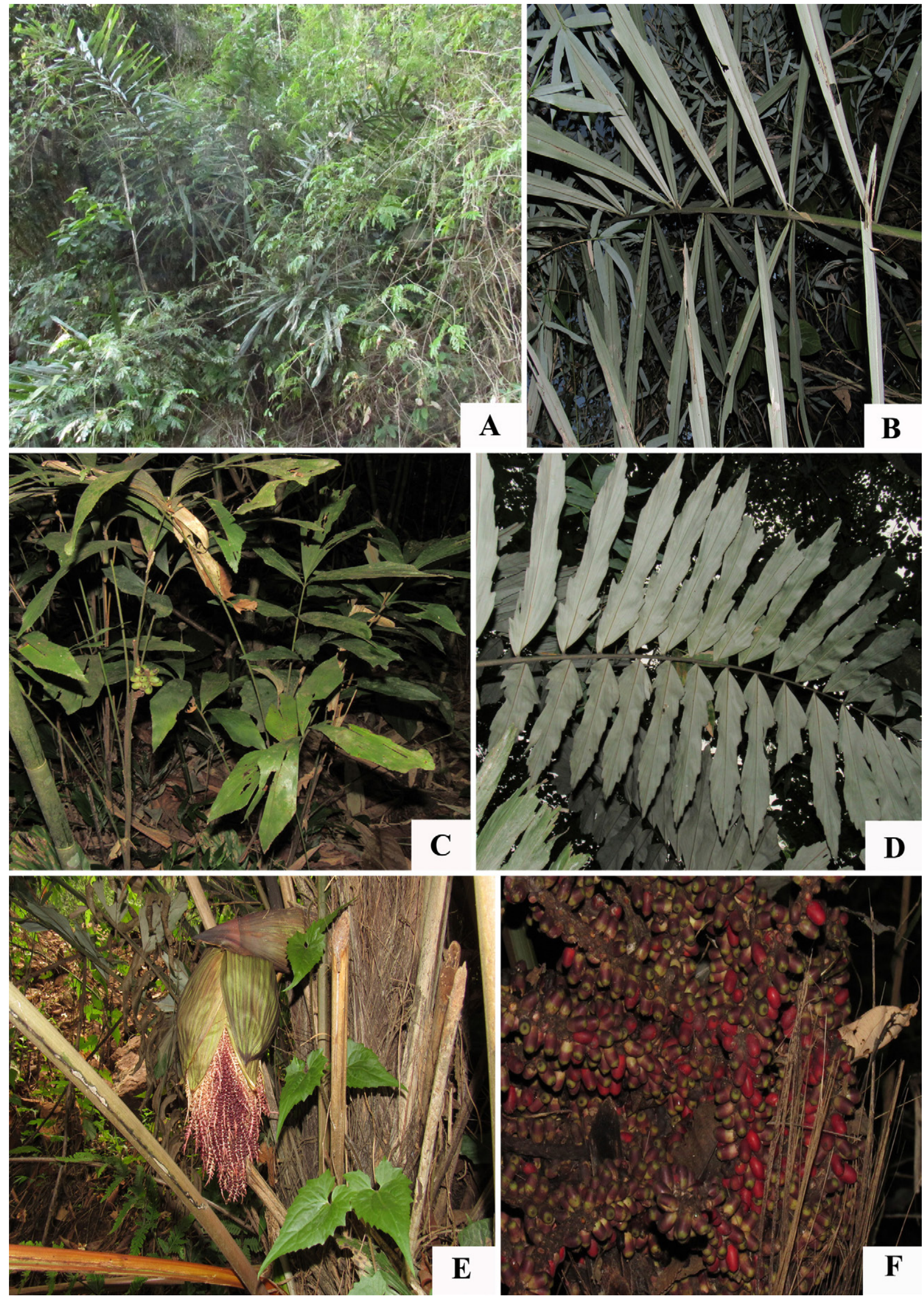

Figure 9. Palms (Arecaceae) in Assam, India. A, B. Wallichia disticha: (A) habit; (B) leaf. C. Wallichia nana C. Habit. D-F. Wallichia oblongifolia: (D) leaf; (E) staminate inflorescence; (F) ripe fruits. 

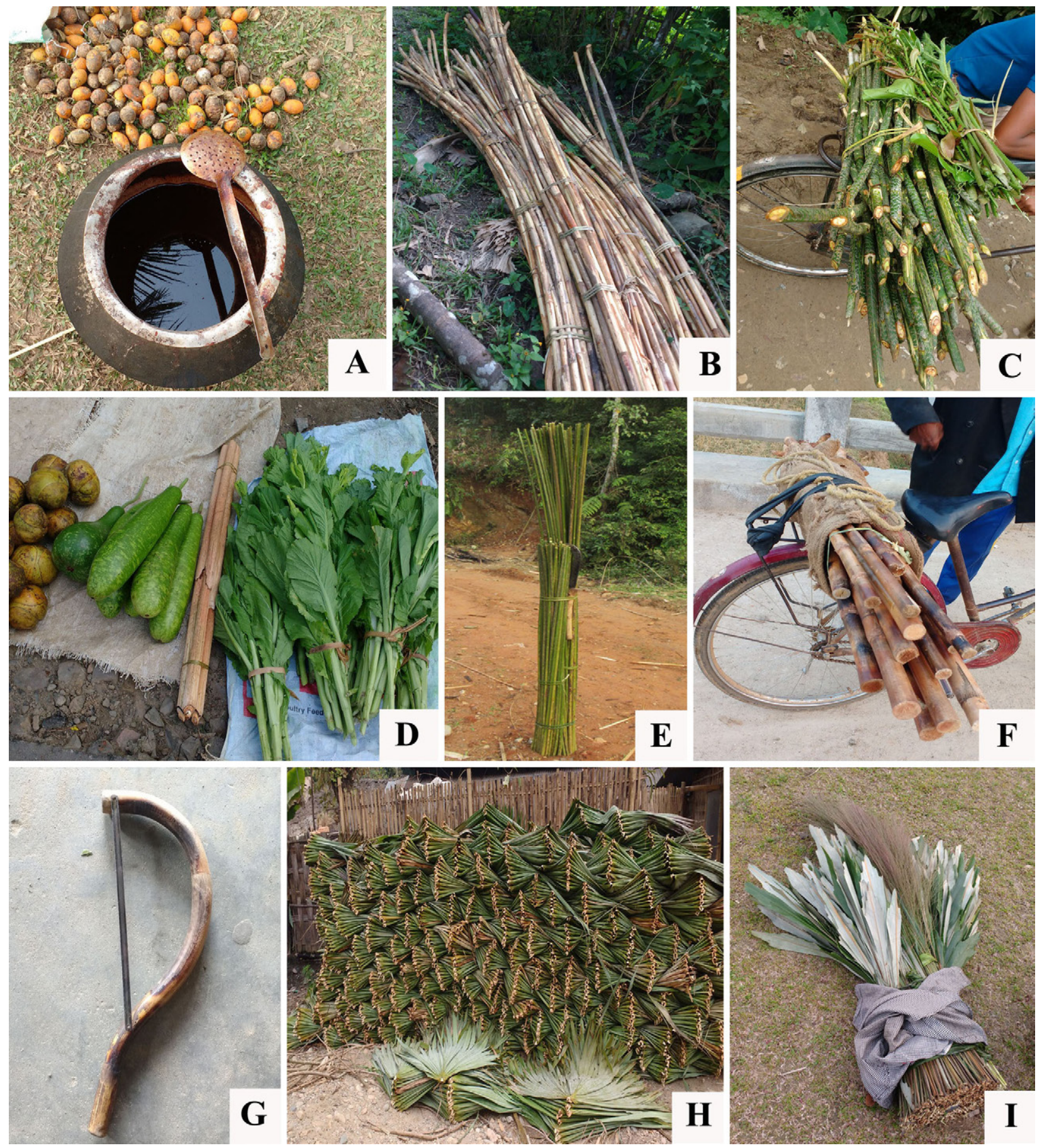

Figure 10. Palms (Arecaceae) in Assam, India. A. Areca catechu. A. Decoction of mature fruits. B. Canes for commercial purposes C. Calamus tenuis. C. Apical shoots as vegetables. D. Calamus flagellum. D. Apical shoots as vegetables. E, F. Calamus melanochaetes: (E) leaf rachises for mat preparation; (F) handle sticks. G. Frame of hacksaw from cane. H. Livistona jenkinsiana commercialization of leaves. I. Wallichia oblongifolia midveins for brooms.

\section{Key to the species of Salacca}

1a Leaflets regular; bract ca $5-7 \mathrm{~cm}$ long

Salacca secunda

1b Leaflets grouped; bract ca $25 \mathrm{~cm}$ long Salacca wallichiana

\section{Salacca secunda Griff.}

Figure 8C, D

Diagnostic characters. Stem acaulescent; leaf pinnate, 4-7 m long; petiole 2-4.2 $\mathrm{m}$ long and $\mathrm{ca} 4 \mathrm{~cm}$ in diameter; spines linear, white, clusters of 5-9, 3-7 cm long.
Leaflets alternate, regular, $76-80 \mathrm{~cm}$ long, ca $5 \mathrm{~cm}$ wide, lanceolate, acuminate, serrated and terminal leaflets connate at base. Staminate inflorescence $1-1.3 \mathrm{~m}$ long; partial inflorescences 6 or 7 in number, $50-90 \mathrm{~cm}$ long; bract 5-7 cm long; rachillae alternate, $11-15 \mathrm{~cm}$ long; staminate flowers dyad, compactly arranged, red, and covered by a bracteole.

Flowering (staminate). September to October.

Distribution. Dibrugarh, Jorhat, East Karbi Anglong, and Tinsukia districts; in and waterlogged plains. 
Vernacular names. Jenglow, Jeng (Assamese).

Uses. For roof (leaf), edible (fruit).

Material examined. INDIA • Mishmi Hills, Glo, Kamlang Valley; alt. 3000-4000 ft; 06 Apr. 1947; F. Kingdom-Ward leg.; NYBG 02320611. • 1 10’; Assam, Jorhat, Mariyani; $26^{\circ} 20^{\prime} 54.5^{\prime \prime} \mathrm{N}, 091^{\circ} 46^{\prime} 02.9^{\prime \prime} \mathrm{E}$; alt. $09 \mathrm{~m}$; 02 Sep. 2018; S. Mehmud leg.; CH, ASSAM 96253.

\section{Salacca wallichiana Mart.}

Figure 8E, F

Diagnostic characters. Stem acaulescent. Leaf pinnate, 4-6 m long; base light yellowish to pink; spines linear, sharp, brown, $2.5-8 \mathrm{~cm}$ long, arrangement irregular. Leaflets in clusters of 3 or 5, 58-60 cm long, 5-6 $\mathrm{cm}$ wide, lanceolate, acuminate. Pistillate inflorescences among the leaf ca $60 \mathrm{~cm}$ long; rachis with brown indumentums; partial inflorescences 5 or 6 in number, 18 $20 \mathrm{~cm}$ long; bracts ca $25 \mathrm{~cm}$ long; rachillae 5-6 cm long, flowers dyad.

Flowering (pistillate). April to May.

Distribution. Jorhat district; in sunny plains.

Vernacular names. Lichu (Assamese).

Uses. Food (fruit).

Material examined. SIAM • Bankok; 21 Jun. 1920; G. W. Groff leg.; NYBG 02320603. INDIA • Assam, Jorhat, Maibeli; $26^{\circ} 42^{\prime} 04.5^{\prime \prime} \mathrm{N}, 094^{\circ} 26^{\prime} 37.4^{\prime \prime} \mathrm{E}$; alt. $60 \mathrm{~m}$; 03 May 2019; S. Mehmud leg.; CH, ASSAM 96254.

\section{Key to the species of Wallichia}

1a Tree; leaflets linear in two planes; fruiting branch ca $2 \mathrm{~m}$, resupinate Wallichia disticha

1b Shrubs; leaflets oblong in one plane; fruiting branch less than $2 \mathrm{~m}$, upward 2

2a Palms ca $1 \mathrm{~m}$ tall; leaflets 4-5 per side; auricle absent Wallichia nana

2b Palms ca $2.5 \mathrm{~m}$ tall; leaflets $30-45$ per side; auricle present Wallichia oblongifolia

\section{Wallichia disticha T. Anderson}

Figure 9A, B

Diagnostic characters. Stems clustered or solitary, woody, unbranched, 6-9 $\mathrm{m}$ tall tree; stem covered by black fibre. Leaf pinnate, alternate, 1.8-3 m long; leaflets paired, arrangement in two planes, 30-60 cm long, 3-5 $\mathrm{cm}$ wide, linear, tip serrated, abaxially silvery white and midvein prominent. Fruiting body ca $2 \mathrm{~m}$ long; rachillae many, 36-42 cm long; fruit ellipsoid, light greenish, $1-1.5 \mathrm{~cm}$ long.

Fruiting. July to August.

Distribution. Cachar district; near streams.

Material examined. INDIA - Assam, Cachar, Borail WLS, Bihara; 09 Oct. 2011; H. A. Borbhuiya leg.; ASSAM 86019. - Assam, Cachar, Kalain; $25^{\circ} 01^{\prime} 34.5^{\prime \prime} \mathrm{N}$, 092 29'43.1"E; alt. 42 m; 13 Jan. 2019; S. Mehmud leg.; CH, ASSAM 96255.

\section{Wallichia nana Griff.}

Figure 9C

Diagnostic characters. Stems clustered, unarmed, erect, ca $1 \mathrm{~m}$ long and $0.8-1.1 \mathrm{~cm}$ in diameter. Leaf pinnate, alternate, 50-70 cm long; ligule fibrous, 7-9 cm long; petiole ca $35 \mathrm{~cm}$. Leaflets regular, oblong, 3 or 4 per side, 20-30 cm long, ca $5 \mathrm{~cm}$ wide, serrated, abaxially silverywhite, terminal leaflets bilobed. Inflorescence terminal, ca $20 \mathrm{~cm}$ long, branched or unbranched; bracts $5-7 \mathrm{~cm}$ long, brown; rachilla 7-12 cm long. Fruits ovate to ellipsoid, light green to white, $1.5-2.2 \mathrm{~cm}$ long.

Flowering and fruiting. August and September to February, respectively.

Distribution. Kamrup Rural, West Karbi Anglong, and Tinsukia districts; prefers moist area.

Material examined. INDIA - Assam, Jorhat, Nambor Reserve Forest; 21 Nov. 1964; S.K. Kataki leg.; ASSAM 44563. • Assam, Tinsukia, Bherjan; 27³1'27.2"N 095²2'14.4"E; alt. 84 m; 03 Sep. 2019; S. Mehmud leg.; CH, ASSAM 96256.

\section{Wallichia oblongifolia Griff.}

Figure 9D, F

Diagnostic characters. Stems solitary or clustered; leaf pinnate, ca $3.5 \mathrm{~m}$ long; petiole ca $2 \mathrm{~m}$ long; indumentums brown. Leaflets regular, alternate, $18-26$ per side, $45-87$ cm long, ca $5 \mathrm{~cm}$ wide, oblong, serrated, abaxially silvery white and terminally 3-4 lobed. Staminate inflorescence resupinate, at the base, ca $75 \mathrm{~cm}$ long; bracts 4 or 5 in number, 20-28 cm long, alternate, ovate; rachillae yellow, ca 50-60 in number, 20-23 cm long; flowers pink to dark purple pink. Pistillate inflorescences erect ca $80 \mathrm{~cm}$ long; rachillae 17-20 in number, 30-48 cm long, green; bracts $24-30 \mathrm{~cm}$ long, linear and ovate; pistillate flowers spirally arranged; few neuter flowers at terminal portion of the rachillae. Fruits ellipsoid, red, 1-1.5 cm long. Flowering and fruiting. April to May and May to November, respectively.

Distribution. Cachar, Dibrugarh, Golaghat, Hailaknadi, Kamrup Metro, Kamrup Rural, East Karbi Anglong, West Karbi Anglong, Kokrajhar and Lakhimpur districts; common in Assam and extend up to $900 \mathrm{~m}$.

Uses. For roofs (leaf), and brooms (midvein).

Material examined. INDIA • Mizoram, Merlan National Park, Pumpet River Forest; 19 Jan. 2013; Ramesh Kumar \& Party leg.; ASSAM 87977. • 1 đ of; Assam, West Karbi Anglong, Nelie Umapanai Khenduli Tapat Road; 26 02'24.9"N, 092 ${ }^{\circ} 16^{\prime} 29.3^{\prime \prime} \mathrm{E}$; alt. 74 m; 06 May 2018; S. Mehmud leg.; CH, ASSAM 96257.

\section{Discussion}

We failed to find three species of Phoenix during our field survey i.e. Phoenix acaulis, $P$. loureiroi, and $P$. rupicola which are reported from Assam (Henderson 2009; Renuka and Sreekumar 2012) but we could not locate voucher specimens in CAL of these three species. 
Nonetheless, one voucher specimen of $P$. acaulis was seen in ASSAM (68797); it had been collected from North Cachar Hills district of Assam. Calamus viminalis was also previously reported from Assam (Barooah and Ahmed 2014), but we did not find any authentic specimens in CAL and ASSAM. Despite our surveys across the Assam, we did not find Phoenix acaulis, P. loureiroi, $P$. rupicola, and $C$. viminalis and these species are excluded from our list.

We document the uses of each species in our list based on our own data collected during the field surveys. Plectocomia assamica and almost all species of Calamus are used for both commercial and domestic purposes. The fruits of Areca catechu, A. triandra, Pinanga gracilis, Calamus tenuis, C. floribundus, C. guruba, C. melanochaetes, Pinanga sylvestris, P. griffithii, Licuala peltata, and Livistona jenkinsiana are used as a masticator. In Kamrup Rural, the decoction of mature nuts of Areca catechu is used to color immature nuts to increase their value (Fig. 10A). Several species of Calamus are used for commercial uses in Cachar district (Fig. 10B). The apical shoots of Calamus tenuis (Fig. 10C), C. flagellum (Fig. 10D), C. leptospadix, C. floribundus, C. nambariensis, and $C$. melanochaetes are used as a vegetable. In Kamrup Rural district, leaf rachises of $C$. melanochaetes is commercially used for mats (Fig. 10E) and stems for handles (Fig. 10 F). The lamina of Livistona jenkinsiana are used for hats and roofs in upper Assam, and its cultivation and commercialization were recorded in Dhemaji district (Fig. 10H). In Lakhimpur district, cane is used for the preparation of hacksaw frames (Fig. 10G) and the Nepali community uses the mid-vein of Wallichia oblongifolia (Fig. 10I) for brooms; the Dimacha community in Dima Hasao district use leaflets of the same species roofs. In addition to the uses in our study, there have been several other reports on uses of Arecaceae members in Assam as medicine (Borah et al. 2006; Saikia et al 2006; Buragohain and Konwar 2007; Sonowal and Baruah 2011; Choudhury et al 2012; Das et al 2013; Borborah et al. 2014; Das and Teron 2014; Deka and Nath 2014; Gogoi and Juji 2015; Ingtipi et al 2016; Dutta et al 2017; Sarkar and Devi 2017), beverage (Pawe and Gogoi 2013), natural dye (Barukiyal and Sarmah 2011), masticator (Borborah et al 2014; Ingtipi et al 2016; Mehmud and Roy 2020b), food (Pandey and Borah 1997; Patir and Borah 2007; Kar et al 2008; Borborah et al 2014; Das and Teron 2014; Medhi et al 2014; Gogoi and Jiji 2015; Dutta et al 2017; Sarkar and Devi 2017), and household (Dutta et al 2017; Henderson 2007).

We found that some wild palms, such as Calamus acanthospathus, C. gracilis, C. henryanus, C. kingianus, C. meghalayensis, and Plectocomia assamica are restricted in their distribution to parts Assam and require conservation efforts. Our study should be helpful with the identification of the palms in Assam and serve to guide future research on palms in the state.

\section{Acknowledgements}

We thank Dr Saranga Ranjan Patgiri (former Head) and Dr Ruma Sarma (present Head) of the Department of Botany, Cotton University, for providing lab facilities; Ranjana Gupta, former Principal Chief Conservator of Forests (Wildlife) and Chief Wildlife Warden, Assam for permission for forest visit (office order no. 341 A dated 2 May 2019 and memo no. WL/FG.31/Pt/Technical Committee/2018); Dr Paramjit Singh, Director of Botanical Survey of India for permission to herbarium visit and library access; Dr Nilakshi Devi, Head of the Department of Botany, Gauhati University for herbarium consultation. We are grateful to several institutions, including the Rain Forest Research Institute (Jorhat, Assam) and the virtual herbaria BR, K, NYBG, and P. We thank Dr Andrew Henderson, New York Botanic Garden, and Jatindra Sarma, Conservator of Forests, Northern Assam Circle for their help and suggestions. We also thank all forests guards and other officials for their assistance. Finally, we thank Saswata Saharia of the Threshold Geospatial Institute (Guwahati) for preparing the distribution map.

\section{Authors' Contributions}

SM conducted field surveys, took the photographs, and collected, identified, preserved, and prepared herbarium specimens, consulted other herbaria, and prepared the manuscript; HR guided the entire work and reviewed the manuscript.

\section{References}

Baishya RA, Sarma J, Begum A (2015) Forest-based medicinal plants rendering their services to the rural community of Assam, India. International Journal of Medicinal Plants Research 4 (1): 314-323.

Barooah C, Ahmed I (2014) Plant diversity of Assam: A checklist of Angiosperms and Gymnosperms. Assam Science Technology and Environment Council, Assam, India, 599 pp.

Barrow SC (1998) A monograph of Phoenix L. (Palmae: Coryphoideae). Kew Bulletin 53 (3): 513-575. https://doi.org/10.2307/4110478

Barukiyal J, Sarmah JN (2011) Ethnomedicinal plants used by people of Golaghat district, Assam, India. International Journal of Medicinal and Aromatic Plants 1 (3): 203-211.

Basu SK (1992) Rattans (Canes) in India: A monographic Revision. Rattan Information Centre, Forest Research Institute Malaysia, Kepong, Malaysia, 141 pp.

Basu SK, Chakraverty RK (1994) A manual of cultivated palms in India. Botanical Survey of India, Calcutta, India, 166 pp.

Beccari O (1908) Asiatic Palms-Lepidocaryeae. Part 1. The species of Calamus. Annals of the Royal Botanic Garden, Calcutta 11: $1-516$.

Beccari O (1911) Asiatic Palms-Lepidocaryeae. Part 2. The species of Daemonorops. Annals of the Royal Botanic Garden, Calcutta 11: $1-237$.

Blatter E (1926) The palms of British India and Ceylon. Oxford University Press, London, UK, 600 pp.

Bora A, Bhattacharyya D (2017) Phytodiversity of Barail Wildlife Sanctuary, Assam, India: field-based observations-I. Trees and lianas. Check List 13 (6): 1037-1053. https://doi.org/10.155 60/13.6.1037

Bora HR, Gogoi G, Kumar R (2012) A systematic census of rattan 
(canes) in Kaziranga National Park, Assam with emphasis of conservation. Journal of Economic and Taxonomic Botany 36 (3): 596-603.

Borah PK, Gogoi P, Phukan AC, Mahanta J (2006) Traditional medicine in the treatment of gastroinstestinal diseases in upper Assam. Indian Journal of Traditional Knowledge 5 (4): 510-512.

Borborah K, Baruah S, Borthakur SK (2014) Plant masticatories and their medicinal importance from Assam \& Meghalaya. International Journal of Herbal Medicine 2 (3): 21-25.

Buragohain J, Konwar BK (2007) Ethnomedicinal plants used in skin diseases by some Indo-Mongoloid communities of Assam. Asian Journal of Experimental Science 21 (2): 281-288.

Choudhury S, Sarma P, Choudhury MD, Sharma GD (2012) Ethnomedicinal plants used by Chorei tribe of Southern Assam, North eastern India. Asian Pacific Journal of Tropical Disease S141S147.

Das AJ, Kumar R, Athar M, Rawat DS, Kumar M, Khan MA (2013) Ethno medicinal study of threatended plants of Sonitpur district of Assam, North East India. International Journal of Pharmacy 4 (1): 146-149.

Das C, Teron R (2014) Ethnobotanical notes of the Rabha community in Mataikhar reserve forest of Kamrup district, Assam, India. Research Journal of Recent Sciences, 3 (6): 26-33.

Deka K, Borthakur SK, Nepi C, Tanti B (2018) Lectotypification of Calamus nambariensis Becc. (Arecaceae). Nordic Journal of Botany 36 (10): e01953. https://doi.org/10.1111/njb.01953

Deka K, Nath N (2014) Application of local health traditional knowledge in oral health and hygiene among the ethnic tribes of Nalbari and Barpeta districts of western Assam (North east India). International Journal of Pure \& Applied Biosciences 2 (5): 107-114.

Dowe JL (2009) A taxonomic account of Livistona R. Br. (Arecaceae). Gardens Bulletin Singapore 60 (2): 185-344.

Dransfield J (1986) A guide to collecting palms. Annals of Missouri Botanical Garden 73 (1): 166-176. https://doi.org/10.2307/2399148

Dutta D, Hazarika P, Hazarika P (2017) Wild edible species in patch vegetation of Jorhat district, Assam, India. International Research Journal of Biological Sciences 6 (3): 14-25.

Dutta D, Hazarika P, Hazarika P (2017) Wild edible species in patch vegetation of Jorhat district, Assam, India. International Research Journal of Biological Sciences 6 (3): 14-25.

Gogoi M, Jiji P (2015) Certain ethno-medicinal plants of Sivsagar district, Assam and their uses. Annals of Pharmacy and Pharmaceutical Sciences 6 (1-2): 26-32.

Griffith W (1845) The palms of British East India. Calcutta Journal of Natural History 5: 1-103, 311-491.

Griffith W (1850) Palms of British East India: posthumous papers bequeathed to the Honourable the East India Company. Periodical Experts Book Agency, Delhi, India, 182 pp.

Henderson A (2007) A revision of Wallichia (Palmae). Taiwania 52 (1) 1-11. https://doi.org/10.6165/tai.2007.52(1).1

Henderson A (2009) Field guide to the palms of southern Asia. Princeton University Press, Princeton, USA, 199 pp.

Henderson A (2020) A revision of Calamus (Arecaceae, Calamoideae, Calameae, Calaminae). Phytotaxa 445 (1): 1-656. https://doi.org/ 10.11646/phytotaxa.445.1.1

Ingtipi W, Teronpi N, Teron R (2016) Ethnomedicinal knowledge for management of leeches in Assam. Defence Life Science Journal 1 (1): 102-106. https://doi.org/10.14429/dlsj.1.10063

Jain SK, Hajra PK (1975) On the botany of Manas Wildlife Sanctuary in Assam. Bulletin of the Botanical Survey of India 17 (1-4) 75-86.

Jain SK, Rao RR (1977) A handbook of field and herbarium methods. Today \& Tomorrow's Printers and Publishers, New Delhi, India, $157 \mathrm{pp}$.

Johnson DV (1998) Non wood forest products, 10. Tropical palms Food and Agriculture Organization of the United Nations, Rome, Italy, 166 pp. http://www.fao.org/3/a-x0451e.pdf. Accessed on: 2017-8-30.
Kanjilal UN, Kanjilal PC, Das A, Dey RN, Bor NL (1934-1940) Flora of Assam. Vols. 1-5. Government of Assam, Shillong, India.

Kar A, Borkotoki S, Borthakur SK (2008) Wild edible fruit of the Karbi's of Karbi Anglong district of Assam, India. Pleione 2 (2): 175181

Medhi P, Sarma A, Borthakur SK (2014) Wild edible plants from the Dima Hasao district of Assam, India. Pleione 8 (1): 133 -148.

Medhi P, Sarma A, Borthakur SK (2014) Wild edible plants from the Dima Hasao district of Assam, India. Pleione 8 (1): 133-148.

Mehmud S, Roy H (2020) A study on flowering pattern to timing of seedling establishment of nine Calamus species from Assam. NeBIO 11 (3): 216-219.

Mehmud S, Roy H (2020a) Henry's Rattan Calamus henryanus Becc. (Arecaceae), a new record to India. Journal of Threatened Taxa 12 (8): 15962-15966. https://doi.org/10.11609/jott.5354.12.8.15962-15966

Mehmud S, Roy H (2020b) Observation of adventitious shoots in three wild palms of Assam, India. Tropical Plant Research 7 (1): 190195. https://doi.org/10.22271/tpr.2020.v7.i1.024

Mehmud S, Roy H (2020c) Anatomical studies on Wallichia nana Griff., a wild palm of Assam, India. Reinwardtia 19 (2): 97-102. https://doi.org/10.14203/reinwardtia.v19i2.3920

Muscarella R, Emilio T, Phillips OL, Lewis SL, Slik F, Baker WJ, Couvreur TLP, Eiserhardt WL, Svenning JC, Affum-Baffoe K, Aiba SI, de Almeida EC, de Almeida SS, de Oliveira EA, Álvarez-Dávila E, Alves LF, Alvez-Valles CM, Carvalho FA, Guarin FA, Andrade A, Aragão LEOC, Murakam AA, Arroyo L, Ashton PS, Corredor GAA, Baker TR, de Camargo PB, Barlow J, Bastin JF, Bengone NN, Berenguer E, Berry N, Blanc L, BöhningGaese K, Bonal D, Bongers F, Bradford M, Brambach F, Brearley FQ, Brewer SW, Camargo JLC, Campbell DG, Castilho CV, Castro W, Catchpole D, Martínez CEC, Chen S, Chhang P, Cho P, Chutipong W, Clark C, Collins M, Comiskey JA, Medina MNC, Costa FRC, Culmsee H, David-Higuita H, Davidar P, del AguilaPasquel J, Derroire G, Fiore AD, Do TV, Doucet JL, Dourdain A, Drake DR, Ensslin A, Erwin T, Ewango CEN, Ewers RM, Fauset S, Feldpausch TR, Ferreira J, Ferreira LV, Fischer M, Franklin J, Fredriksson GM, Gillespie TW, Gilpin M, Gonmadje C, Gunatilleke AUN, Hakeem KR, Hall JS, Hamer KC, Harris DJ, Harrison RD, Hector A, Hemp A, Herault B, Pizango CGH, Coronado ENH, Hubau W, Hussain MS, Ibrahim FH, Imai N, Joly CA, Joseph S, K A, Kartawinata K, Kassi J, Killeen TJ, Kitayama K, Klitgård BB, Kooyman R, Labrière N, Larney E, Laumonier Y, Laurance SG, Laurance WF, Lawes MJ, Levesley A, Lisingo J, Lovejoy T, Lovett JC, Lu X, Lykke AM, Magnusson WE, Mahayani NPD, Malhi Y, Mansor A, Peña JLM, Marimon-Junior BH, Marshall AR, Melgaco K, Bautista CM, Mihindou V, Millet J, Milliken W, Mohandass D, Mendoza ALM, Mugerwa B, Nagamasu H, Nagy L, Seuaturien N, Nascimento MT, Neill DA, Neto LM, Nilus R, Vargas MPN, Nurtjahya E, de Araújo RNO, Onrizal O, Palacios WA, Palacios-Ramos S, Parren M, Paudel E, Morandi PS, Pennington RT, Pickavance G, III JJP, Pitman NCA, Poedjirahajoe E, Poorter L, Poulsen JR, Prasad PRC, Prieto A, Puyravaud JP, Qie L, Quesada CA, Ramírez-Angulo H, Razafimahaimodison JC, Reitsma JM, Requena-Rojas EJ, Correa ZR, Rodriguez CR, Roopsind A, Rovero F, Rozak A, Lleras AR, Rutishauser E, Rutten R, Punchi-Manage R, Salomão RP, Sam HV, Sarker SK, Satdichanh M, Schietti J, Schmitt CB, Marimon BS, Senbeta F, Sharma LN, Sheil D, Sierra R, Silva-Espejo JE, Silveira M, Sonké B, Steininger MK, Steinmetz R, Stévart T, Sukumar R, Sultana A, Sunderland TCH, Suresh HS, Tang T, Tanner E, Steege HT, Terborgh JW, Theilade I, Timberlake T, TorresLezama A, Umunay P, Uriarte M, Gamarra LV, van de Bult M, van der Hout P, Martinez RV, Vieira ICG, Vieira SA, Vilanova E, Cayo JV, Wang O, Webb CO, Webb EL, White L, Whitfeld TJS, Wich S, Willcock W, Wiser SK, Young KR, Zakaria Z, Zang R, Zartman CE, Zo-Bi IC, Balslev H (2020) The global abundance of tree palms. Global Ecology \& Biogeography 29: 1495-1514. https://doi.org/10.1111/geb.13123 
Palm Web (2019) http://palmweb.org. Accessed on: 2019-6-4.

Pandey Ak, Bora HR (1997) Edible plants of Shan tribe of Assam. Ancient Science of Life 16 (4): 258-276.

Patir B, Borah A (2007) Wild edible plants of Assam. Geetakhi Printers \& Publishers, Guwahati, India, 21 pp.

Pawe D, Gogoi R (2013) Ethnobotany of Poro Apong or Chai Mod- A local rice beer of Mishing tribes of Assam. NeBio 4 (2): 46-49.

Qureshimatva Q, Gamit SB, Solanki HA (2020) Arecaceae family in Gujrat, its morphological character based identification keys. India Forester 146 (2): 113-124.

Rahman H (2007) Diversity of rattans canes in Assam and their conservation. Journal of Economic and Taxonomic Botany 31(4): 907 912.

Rao AS, Verma DM (1970) Materials towards a monocot flora of Assam (Hydrocharitaceae and Burmanniaceae). Bulletin of the Botanical Survey of India 12 (1-4): 139-143.

Rao AS, Verma DM (1972) Materials towards a monocot flora of Assam II (Zingiberaceae and Marantaceae). Bulletin of the Botanical Survey of India 14 (1-4): 114-143.

Rao AS, Verma DM (1973) Materials towards a monocot flora of Assam III (Taccaceae, Dioscoreaceae and Stemonaceae). Bulletin of the Botanical Survey of India 15 (3-4): 189-203.

Rao AS, Verma DM (1974) Materials towards a monocot flora Assam IV (Pontederiaceae, Xyridaceae \& Commelinaceae). Bulletin of the Botanical Survey of India 16 (1-4): 1-20.

Rao AS, Verma DM (1976) Materials towards a monocot flora Assam
V. Botanical Survey of India 18 (1-4): 1-48.

Renuka C, Bhat KV, Pandalai RC (2010) Rattans of India-taxonomy, biology and utilization. Kerala Forest Research Institute, Thrissur, Kerala, India, 339 pp.

Renuka C, Shreekumar VB (2012) A field guide to the palms of India. Kerala Forest Research Institute, Peechi, Thrissure, Kerala, India, $256 \mathrm{pp}$.

Saikia AP, Ryakala VK, Sharma P, Goswami P, Bora U (2006) Ethnobotany of medicinal plants used by Assamese people for various skin ailments and cosmetics. Journal of Ethnopharmacology 106: 149-157. https://doi.org/10.1016/j.jep.2005.11.033

Sarkar M, Devi A (2017) Analysis of medicinal and economic important plant species of Hollongapar Gibbon Wildlife sanctuary Assam, northeast India. Tropical Plant Research 4 (3): 486-495. https://doi.org/10.22271/tpr.2017.v4.i3.065

Sonowal R, Baruah I (2011) Ethnomedical practices among the TaiKhamyangs of Assam, India. Ethno Med 5 (1): 41-50.

The Plant List (2020) www.theplantlist.org. Accessed on: 2020-2-7.

van Rheede tot Draakestein H (1678) Hortus Indicus Malabaricus. Continens Regni Malabarici apud Indos celeberrimi omnis generis Plantas rariores, Latinis, Malabaricis, Arabicis \& Bramanum Characteribus nomibusque expressas, Una cum Floribus, Fructibus \& feminibus, naturali magnitudine a peritiffimispictoribus delineates \&ad vivum exhibitus. Vol. 1. Johannis van Someren, et Joannis van Dyck, Amsterdam, 110 pp., 56 pls. https://doi. org/10.5962/bhl.title.707 